\title{
Quench dynamics of one-dimensional bosons in a commensurate periodic potential: A quantum kinetic equation approach
}

\author{
Marco Tavora and Aditi Mitra \\ Department of Physics, New York University, 4 Washington Place, New York, New York 10003, USA
}

(Dated: September 17, 2018)

\begin{abstract}
Results are presented for the dynamics arising due to a sudden quench of a boson interaction parameter with the simultaneous switching on of a commensurate periodic potential, the latter providing a source of non-linearity that can cause inelastic scattering. A quantum kinetic equation is derived perturbatively in the periodic potential and solved within the leading order gradient expansion. A two-particle irreducible formalism is employed to construct the stress-momentum tensor and hence the conserved energy. The dynamics is studied in detail in the phase where the boson spectrum remains gapless. The periodic potential is found to give rise to multi-particle scattering processes that relaxes the boson distribution function. At long times the system is found to thermalize with a thermalization time that depends in a non-monotonic way on the amount of energy injected into the system due to the quantum quench. This non-monotonic behavior arises due to the competing effect of an increase of phase space for scattering on the one hand, and an enhancement of the orthogonality catastrophe on the other hand as the quench amplitude is increased. The approach to equilibrium is found to be purely exponential for large quench amplitudes, and more complex for smaller quench amplitudes.
\end{abstract}

PACS numbers: 05.70.Ln,37.10.Jk,71.10.Pm,03.75.Kk

\section{INTRODUCTION}

How a many particle system prepared in an initial state which is far from equilibrium evolves in time, and how it thermalizes if at all, is an important fundamental question which is also of experimental relevance due to the realization of ideal, thermally isolated, quantum systems using ultra-cold atomic gases $\underline{1}$ The issue of thermalization becomes all the more important for one-dimensional (1D) and quasi 1D systems where phase space for scattering is so highly restrictive that even generic systems with no special conservation laws can show enormous bottlenecks to thermalization. ${ }^{2-4}$ In fact in the past, classical 1D systems were also found to show a bottleneck to thermalization, $\frac{5}{5}$ where a quantitative explanation for this was found to be quite subtle, requiring an understanding of integrability, the Kolmogorov-Arnold-Moser (KAM) theorem and chaos.

One appealing way to understand thermalization or the lack of it in quantum systems is to borrow concepts from classical systems. In particular the lack of thermalization in many $1 \mathrm{D}$ systems has been attributed to these systems being similar to ideal integrable models $\stackrel{2}{2}$ Integrable models have the property that they have many more conserved quantities besides energy and particle number, $\underline{6}$ which strongly restricts their dynamics. However the exact effect of integrability on dynamics is not understood in any quantitative way as the notion of integrability cannot be generalized to quantum systems rigorously because classical and quantum systems differ in the way the number of degrees of freedom are counted ${ }^{7}$ This leads to confusion as to how to collect all the relevant integrals of motion, and how many of these need to be kept in order to understand the timeevolution and the long time behavior $\stackrel{8}{-}$ Recent studies have proposed keeping only the most "local" integrals of motion. ${ }^{9-11}$ These selective integrals of motion have been used to construct generalized Gibbs ensembles (GGEs) 12 with the aim to understand the long time steady-state behavior after a quantum quench. However understanding the temporal evolution from the initial state to the state described by the GGE in integrable models, in particular those described by interacting field theories is a challenging issue that has been addressed only recently for the time-evolution of some single-particle expectation values, ${ }^{13}-15$ in contrast how two-point and higher order correlations evolve in time, and whether their behavior can be captured by a GGE is largely unexplored.

Numerical studies on finite size systems have also been extensively carried out to understand the issue of thermalization. The two main approaches used are exact diagonalization ${ }^{16-20}$ and integrability based methods. ${ }^{21}$ Some of these studies ${ }^{16,17}$ indicate that thermalization is consistent with the eigenstate-thermalization-hypothesis (ETH),$\stackrel{22,23}{2}$ and is related to the level statistics of the Hamiltonian. ${ }^{20}$ However it is a challenging task to generalize these results to systems in the thermodynamic limit.

Perhaps one of the most powerful methods to study dynamics of many-particle systems in the thermodynamic limit is the Schwinger-Keldysh or the quantum Boltzmann equation approach. 24,25 While the success of this approach relies on the existence of a small parameter which allows one to truncate the self-energy to a finite order, it is still a non-perturbative approach. These methods have been used to study nonequilibrium dynamics in a variety of interacting fermionic and bosonic field theories, revealing thermalization for some cases, ${ }^{26-30}$ and the appearance of intriguing non-thermal quasi-stationary steady-states for other cases,,$^{30}-34$ where some of these 
steady-states have been related to turbulence $\frac{35}{3}$

A promising direction of research is to employ a quantum kinetic equation to study the dynamics of integrable models, and to then study how integrability breaking terms affect the results. Recently such a study was undertaken for the fermionic Hubbard model with nearest-neighbor ${ }^{36}$ and next-nearest-neighbor ${ }^{37}$ interactions, where it was shown that the kinetic equation for the integrable case (nearest-neighbor interactions) allows for an infinite number of athermal steady-states besides the thermal steady-state corresponding to the Fermidistribution function. Whether a system ends up in one of these athermal steady-states or the thermal state depends on the initial condition. Detailed studies also exist on how a $1 \mathrm{D}$ system weakly perturbed from an initial thermal equilibrium state relaxes to equilibrium, $\underline{\underline{38}-\underline{40}}$ where the weak deviation from thermal equilibrium allows for a linearization of the Boltzmann equation. However such a linearization procedure cannot be applied to quantum quenches since a quench always generates a highly nonequilibrium distribution function.

The aim of the current paper is to study quench dynamics employing a quantum kinetic equation approach. In particular we study a 1D system of interacting bosons in a commensurate periodic potential. We envision a situation where the 1D bosons are initially in the continuum (thus no periodic potential). Using Feshbach resonance techniques, at an initial time $t=0$, the interaction strength of the bosons is suddenly quenched driving them out of equilibrium. A commensurate periodic potential is also switched on at the same time as the interaction quench. A periodic potential gives rise to Umpklapp or backscattering which can relax the distribution function of the bosons, however its effect has never been studied before using a quantum kinetic equation approach. In this paper we plan to fill this gap. In particular we employ a bosonization prescription to study the system, where the bosons in a commensurate periodic potential is described by the sine-Gordon model $\stackrel{41}{ }$ Bosonization allows us to treat the effect of forward scattering between bosons exactly, while the quantum kinetic equation is derived perturbatively in the Umpklapp or backscattering processes.

The ground state of bosons in a commensurate periodic potential has two possible phases $\underline{\underline{41}}$ one is the superfluid phase where the periodic potential is an irrelevant perturbation, and the low-energy theory of the bosons is described by the Luttinger liquid. The second phase is the Mott insulator where the periodic potential is relevant and localizes the bosons. We will be interested in studying quenches within the superfluid phase where the periodic potential is irrelevant in equilibrium. However out of equilibrium, irrelevant terms cannot be dropped $\stackrel{42}{2}$ and can eventually thermalize a system. We plan to study in detail how thermalization comes about due to Umpklapp processes. Of course, other irrelevant terms such as band curvature are also present, however in this paper we retain only the irrelevant term corresponding to the commensurate periodic potential. Keeping more than one irrelevant term is technically challenging, and beyond the scope of this paper. Moreover, for certain quench parameters that we study, the cosine potential is the leading irrelevant operator, and therefore its effects (such as inelastic scattering rate) will dominate over other irrelevant operators.

Note that a periodic potential in a bosonization language is highly nonlinear in the bosonic fields as it is given by $g \cos (2 \phi)$, where $-\partial_{x} \phi / \pi$ is the boson density. Since the system is a superfluid, $\phi$ is highly fluctuating, and the quench only increases these fluctuations. Thus we are not allowed to Taylor expand the $\cos (2 \phi)$ term. Neither are semi-classical approaches such as the Truncated Wigner Approximation valid $\stackrel{43.44}{=}$ Instead we will derive a quantum kinetic equation perturbatively in the strength of the periodic potential $g$. In doing so we will show that the periodic potential leads to an unusual kinetic equation that even to $\mathcal{O}\left(g^{2}\right)$ leads to multi-particle scattering of bosons. This is in marked contrast to studies on $\phi^{4}$ theory or fermionic models where the leading order terms in the kinetic equation capture two-particle $\frac{36,37}{}$ or three-particle scattering processes $\underline{39.45}$

In this paper we present results for the dynamics of the 1D sine-Gordon model in its gapless phase using a quantum kinetic equation approach. In contrast, in the gapped phase, the perturbative expansion in $g$ employed to derive the kinetic equation is not valid. The sine-Gordon model is the continuum limit of the BoseHubbard model which is non-integrable. At the same time, the sine-Gordon model is also the continuum limit of the nearest-neighbor XXZ spin-chain which is integrable. The continuum model is some approximation of the lattice model and does not share all its features. While the sine-Gordon model in its gapped phase is

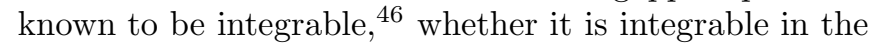
gapless phase is not a question straightforward to address. The reason is that the sine-Gordon model in the gapless phase has ultra-violate (UV) divergences that need to be regularized, and the integrability or lack of it may very well depend on the short-distance physics and therefore the regularization scheme. In this paper we follow a particular regularization scheme, and the final results, once expressed in terms of suitable dimensionless units, does not depend on this regularization. However since we always see thermalization, we think that our model does not have any non-trivial conservation laws. Thus our results are probably not relevant to the integrable XXZ chain, and are more relevant for the non-integrable Bose-Hubbard model. However the precise connection between the results of this paper, and the integrability or lack of it of the original lattice models needs further exploration.

This paper is organized as follows. In Section [I we present the model that will be studied, and briefly discuss the properties of the quench in the absence of the periodic potential where the system reduces to the exactly solvable Luttinger model. In Section [II the Dyson equa- 
tion to leading non-trivial order in the periodic potential is derived, and simplified using a leading order gradient expansion. In Section IV a two-particle irreducible (2PI) formalism is used to show that the Dyson equation can equivalently be derived from a variational approach applied to the 2PI action. We show that this approach also naturally leads to the derivation of a stress-momentum tensor, and from that the conserved energy. In Section $V$ we present our results for the time-evolution of the boson distribution function to leading order in the gradient expansion. Here we discuss time-scales for thermalization and present an analytic estimate for this time-scale. Finally in Section VI we present our conclusions. Some of the details of the calculations are relegated to Appendices $\mathrm{A}, \mathrm{B}$ and $\mathrm{C}$.

\section{MODEL}

The Hamiltonian for interacting bosons in a periodic potential after bosonization is given by,

$$
\begin{aligned}
H & =H_{0}+V_{\mathrm{sg}} \\
H_{0} & =\frac{u}{2 \pi} \int d x\left\{K[\pi \Pi(x)]^{2}+\frac{1}{K}\left[\partial_{x} \phi(x)\right]^{2}\right\} \\
V_{\mathrm{sg}} & =-\frac{g u}{\alpha^{2}} \int d x \cos (\gamma \phi(x))
\end{aligned}
$$

where $H_{0}$ is the quadratic part which describes the Luttinger liquid or long lived sound modes that propagate with velocity $u$. The density of the sound modes is $\rho=$ $-\partial_{x} \phi / \pi$, whereas $\Pi=\partial_{x} \theta / \pi$ is the variable canonically conjugate to $\phi,\left[\phi, \partial_{y} \theta(y)\right]=i \pi \delta(x-y) . V_{\text {sg }}$ represents the commensurate periodic potential whose most important effect is a source of backscattering which can localize the density modes via the well known BerezenskiiKosterlitz-Thouless (BKT) transition. ${ }^{41}$ Note that we will use the convention that for bosons

$$
\gamma=2
$$

while $K=1$ corresponds to hard-core bosons, and $K \rightarrow$ $\infty$ represents non-interacting bosons. In this notation, the critical point separating the Mott-insulating phase from the superfluid phase, in the limit $g \rightarrow 0$, is located at $K=2$. In a renormalization group language, the cosine potential is a relevant perturbation for $K<2$, and irrelevant otherwise, provided $g$ is sufficiently small. In this paper we will be concerned with quench dynamics within the superfluid phase.

It is convenient to represent the fields $\phi, \theta$ in terms of bosonic creation and annihilation operators $\left(b_{p}, b_{p}^{\dagger}\right), 41$

$$
\begin{aligned}
& \phi(x)=-\left(N_{R}+N_{L}\right) \frac{\pi x}{L} \\
& -\frac{i \pi}{L} \sum_{p \neq 0}\left(\frac{L|p|}{2 \pi}\right)^{1 / 2} \frac{1}{p} e^{-\alpha|p| / 2-i p x}\left(b_{p}^{\dagger}+b_{-p}\right) \\
& \theta(x)=\left(N_{R}-N_{L}\right) \frac{\pi x}{L} \\
& +\frac{i \pi}{L} \sum_{p \neq 0}\left(\frac{L|p|}{2 \pi}\right)^{1 / 2} \frac{1}{|p|} e^{-\alpha|p| / 2-i p x}\left(b_{p}^{\dagger}-b_{-p}\right) .
\end{aligned}
$$

where

$$
\Lambda=u \alpha^{-1}
$$

is an ultra-violet cutoff. Thus,

$$
H_{0}=\sum_{p \neq 0} u|p| \eta_{p}^{\dagger} \eta_{p}
$$

where $\eta$ and $b$ are related by the canonical transformation,

$$
\begin{aligned}
\eta_{p} & =\cosh \beta_{1} b_{p}+\sinh \beta_{1} b_{-p}^{\dagger} \\
\eta_{-p}^{\dagger} & =\cosh \beta_{1} b_{-p}^{\dagger}+\sinh \beta_{1} b_{p} .
\end{aligned}
$$

and $e^{-2 \beta_{1}}=K, u=v_{F} / K$.

\section{A. Interaction quench in the Luttinger Model: Properties of the quadratic theory}

In this sub-section, let us assume that $g=0$, so that we have an exactly solvable theory, namely the Luttinger model which captures the characteristic power-law decays of the Luttinger liquid. In equilibrium, the Luttinger liquid is an approximate low-energy description of more complicated models in their gapless phases such as the XXZ spin-chain and the Bose-Hubbard model. Out of equilibrium, when $g \neq 0$, there is no reason to expect that the effective low energy or long wavelength theory is described by the Luttinger liquid ${ }^{42}$ as irrelevant operators cause inelastic scattering. The aim of this paper is to explore these effects.

Dynamics arising either due to a sudden quench or due to more complicated time-dependent protocols have been studied extensively in the literature for the Luttinger model ${ }^{47-52}$ Here we present some results that we will need for the $g \neq 0$ case. Let us suppose that the system at time $t<0$ is a Luttinger liquid with interaction parameter $K_{0}$ and velocity $u_{0}$, and therefore described by the Hamiltonian

$$
\begin{aligned}
& H_{i}=\frac{u_{0}}{2 \pi} \int d x\left\{K_{0}[\pi \Pi(x)]^{2}+\frac{1}{K_{0}}\left[\partial_{x} \phi(x)\right]^{2}\right\} \\
& =\sum_{p \neq 0} u_{0}|p| \eta_{p}^{\dagger} \eta_{p}
\end{aligned}
$$


Let us suppose that at $t=0$ there is an interaction quench from $K_{0} \rightarrow K$ so that the time evolution from $t>0$ is due to

$$
\begin{aligned}
& H_{f}=\frac{u}{2 \pi} \int d x\left\{K[\pi \Pi(x)]^{2}+\frac{1}{K}\left[\partial_{x} \phi(x)\right]^{2}\right\} \\
& =\sum_{p \neq 0} u|p| \gamma_{p}^{\dagger} \gamma_{p}
\end{aligned}
$$

To simplify the algebra we make the assumption that the quench preserves Galilean invariance $u=v_{F} / K, u_{0}=$ $v_{F} / K_{0}$. In the language of the Luttinger model this is equivalent to always having $g_{2}$ and $g_{4}$ processes of the same magnitude $\stackrel{41}{\underline{1}}$ Note that the following canonical transformation relate the different sets of bosons,

$$
\begin{aligned}
\left(\begin{array}{c}
b_{p} \\
b_{-p}^{\dagger}
\end{array}\right) & =\left(\begin{array}{cc}
\cosh \beta_{1} & -\sinh \beta_{1} \\
-\sinh \beta_{1} & \cosh \beta_{1}
\end{array}\right)\left(\begin{array}{c}
\gamma_{p} \\
\gamma_{-p}^{\dagger}
\end{array}\right) \\
\left(\begin{array}{c}
b_{p} \\
b_{-p}^{\dagger}
\end{array}\right) & =\left(\begin{array}{cc}
\cosh \beta_{0} & -\sinh \beta_{0} \\
-\sinh \beta_{0} & \cosh \beta_{0}
\end{array}\right)\left(\begin{array}{c}
\eta_{p} \\
\eta_{-p}^{\dagger}
\end{array}\right)
\end{aligned}
$$

where $e^{-2 \beta_{0}}=K_{0}, e^{-2 \beta_{1}}=K$.

Let us define the functions

$$
\begin{aligned}
f(p t) & =\cos (u|p| t) \cosh \beta_{0} \\
& -i \sin (u|p| t) \cosh \left(2 \beta_{1}-\beta_{0}\right) \\
g(p t) & =\cos (u|p| t) \sinh \beta_{0} \\
& +i \sin (u|p| t) \sinh \left(2 \beta_{1}-\beta_{0}\right)
\end{aligned}
$$

These functions determine the time-evolution after the quench $(t>0)$,

$$
\begin{aligned}
b_{p}^{\dagger}(t)+b_{-p}(t) & =\left(f^{*}(p t)-g(p t)\right) \eta_{p}^{\dagger}(0) \\
& +\left(f(p t)-g^{*}(p t)\right) \eta_{-p}(0) \\
b_{p}^{\dagger}(t)-b_{-p}(t) & =\left(f^{*}(p t)+g(p t)\right) \eta_{p}^{\dagger}(0) \\
& -\left(f(p t)+g^{*}(p t)\right) \eta_{-p}(0)
\end{aligned}
$$

Note that the dynamics couples only the $q,-q$ modes but there is no scattering between modes of different $|q|$. This will change when the periodic potential is applied in a way that will be discussed in detail later. Moreover the dynamics is always translationally invariant in position, both in the absence and presence of the periodic potential as long as we are in the weak-coupling (in $g$ ) regime where the perturbative treatment of this paper is valid. Of course looking for the growth of spatial instabilities after a quench is also an interesting direction of study, $\underline{53}$ which we do not address here.

We will find it convenient to define the following two exponents,

$$
\begin{array}{r}
K_{\text {eq }}=\frac{\gamma^{2}}{4} K \\
K_{\text {neq }}=\frac{\gamma^{2}}{8} K_{0}\left(1+\frac{K^{2}}{K_{0}^{2}}\right)
\end{array}
$$

In terms of these exponents the critical point separating the gapped and the gapless phases in the limit $g \rightarrow 0$ is located at $K_{\mathrm{eq}}=2$. In the ground state of the final
Hamiltonian, correlators such as $\left\langle e^{i \gamma \phi(1)} e^{-i \gamma \phi(2)}\right\rangle$ decay as a power-law determined by $K_{\text {eq }}$, whereas at long times after the quench, the very same correlators decay as a

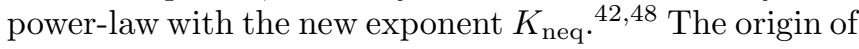
these new exponents is the underlying integrability of the Luttinger model where the boson occupation number for each $q$ is conserved. A more quantitative way to understand why after a quench the decays are still power-law is by noticing that the quench results in a mode-occupation

$$
n(q)=\left\langle\gamma_{q}^{\dagger} \gamma_{q}\right\rangle=\frac{\left(K_{0}-K\right)^{2}}{4 K K_{0}}=\frac{1}{2}\left(\frac{K_{\mathrm{neq}}}{K_{\mathrm{eq}}}-1\right)
$$

which is plotted in Fig. 1. This nonequilibrium distribution may be interpreted as one where there is a momentum or mode dependent temperature. However since $n(q=0)$ is finite, it implies that the momentum dependent temperature vanishes for long wavelengths. Since the power-law or lack of it is primarily determined by the mode-occupation or the effective temperature of the long wavelength modes, the power law survives in the Luttinger model after a quench, though the new exponent $K_{\text {neq }}$ determines the decay. As we show below, the fractional change in the decay exponent $\left[K_{\text {neq }}-K_{\text {eq }}\right] / K_{\text {eq }}$ is a measure of how far out of equilibrium the system is driven due to the quantum quench.

It is straightforward to see that the the modeoccupation in Eq. (21) implies the following energy per unit length of the system,

$$
\begin{aligned}
& \frac{E}{L}=\frac{1}{L} \sum_{p \neq 0} e^{-\alpha|p|} u|p|\left\langle\gamma_{p}^{\dagger} \gamma_{p}\right\rangle=\frac{u}{4 \pi \alpha^{2}} \frac{\left(K_{0}-K\right)^{2}}{K K_{0}} \\
& =\frac{u}{2 \pi \alpha^{2}}\left(\frac{K_{\text {neq }}}{K_{\text {eq }}}-1\right)
\end{aligned}
$$

Obviously the energy injected into the system depends on the underlying lattice cut-off $u / \alpha$. However once length and energies are expressed in units of this cut-off, we have a cut-off independent result for the energy injected into the system,

$$
\frac{\alpha E / u}{L / \alpha}=\frac{1}{2 \pi}\left(\frac{K_{\text {neq }}}{K_{\text {eq }}}-1\right)
$$

Thus the energy injected into the system due to the quench is proportional to the fractional change in the exponent governing the power-law decay at long times, $\left[K_{\text {neq }}-K_{\text {eq }}\right] / K_{\text {eq }}$. This is an important energy scale which will determine the inelastic scattering rate and therefore the thermalization time when the cosine potential is switched on.

In the presence of non-linearities and at long times, the system is expected to eventually thermalize to the equilibrium (mode independent) temperature $T_{\text {eq }}$. We now express this temperature in terms of the quench parameters $K_{\text {eq }}$ and $K_{\text {neq }}$. Using $\left\langle\gamma_{p}^{\dagger} \gamma_{p}\right\rangle=\frac{1}{e^{u|p| / T_{\text {eq }}-1}}$, the energy density at thermal equilibrium is,

$$
\frac{E}{L}=\frac{u}{2 \pi}\left[\frac{2 T_{\mathrm{eq}}}{u^{2}} \psi^{\prime}\left(\frac{\alpha T_{\mathrm{eq}}}{u}\right)-\frac{2}{\alpha^{2}}\right]
$$




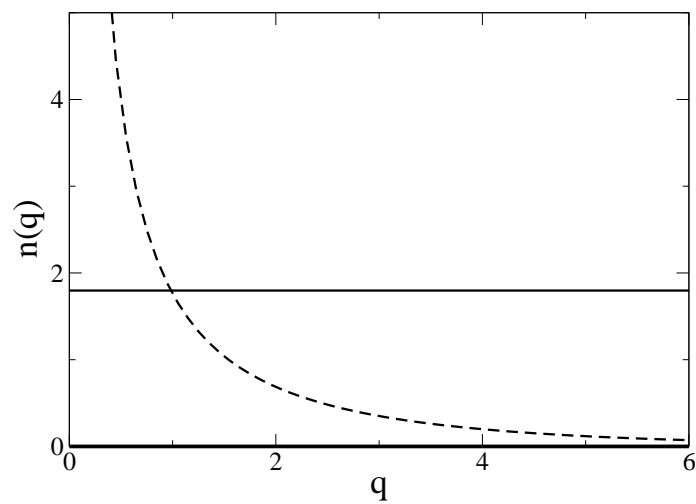

FIG. 1. The boson distribution function $n(q)$ generated by an interaction quench where $K_{\text {neq }}=13.8$ and $K_{\text {eq }}=3$ is $n(q)=\frac{1}{2}\left(\frac{K_{\text {neq }}}{K_{\text {eq }}}-1\right) \approx 1.797$ (continuous line). This is compared with the boson distribution at equilibrium at a non-zero temperature $1 /\left[e^{u|q| / T_{\mathrm{eq}}}-1\right]$, where $T_{\mathrm{eq}}=2.23$ is the temperature associated with the energy injected into the system due to the quench (dashed line). At zero temperature equilibrium, $n(q) \equiv 0$.

where $\psi(z)=\frac{\Gamma^{\prime}(z)}{\Gamma(z)}$ and $\Gamma(z)$ is the gamma function. Since the system is closed, energy conservation implies that Eq. (22) and Eq. (24) are equal to each other, so that $T_{\mathrm{eq}}$ is related as follows to the quench parameters,

$$
\begin{aligned}
& \frac{E}{L}=\frac{u}{2 \pi}\left[\frac{2 T_{\mathrm{eq}}}{u^{2}} \psi^{\prime}\left(\frac{\alpha T_{\mathrm{eq}}}{u}\right)-\frac{2}{\alpha^{2}}\right] \\
& =\frac{u}{2 \pi \alpha^{2}}\left(\frac{K_{\mathrm{neq}}}{K_{\mathrm{eq}}}-1\right)
\end{aligned}
$$

Taking the limit of high temperatures, Eq. (25) becomes, $\frac{E}{L} \approx-\frac{u}{\pi \alpha^{2}}+\frac{T_{\mathrm{eq}}}{\pi \alpha}$. For high temperatures, $\frac{\alpha}{u} T_{\mathrm{eq}} \gg$ 1 (or large quenches $K_{\text {neq }} \gg K_{\text {eq }}$ ) energy conservation implies,

$$
\frac{\alpha}{u} T_{\mathrm{eq}} \approx \frac{1}{2} \frac{K_{\mathrm{neq}}}{K_{\mathrm{eq}}}
$$

For small quenches $\left(K_{\text {neq }}-K_{\text {eq }}\right) / K_{\text {eq }} \rightarrow 0$, and hence low temperatures, the equilibrium temperature is given by,

$$
\frac{\alpha}{u} T_{\mathrm{eq}} \approx \frac{\sqrt{3}}{\pi} \sqrt{\frac{K_{\mathrm{neq}}}{K_{\mathrm{eq}}}-1}
$$

Fig. 1 shows the distribution function $n(q)=\left\langle\gamma_{q}^{\dagger} \gamma_{q}\right\rangle$ generated by an interaction quench and compares it with the thermal distribution at temperature $T_{\text {eq }}$. For the Luttinger model, $n(q)$ is stable in time as there are no relaxation mechanisms. The aim of this paper is to understand how the periodic potential relaxes the distribution function, and whether it ever reaches the thermal distribution shown by the dashed line in Fig. 1.

\section{PERIODIC-POTENTIAL: DERIVATION OF THE DYSON EQUATION}

We now turn to the case where a periodic potential is also present after the quench. Thus the Hamiltonian at $t \leq 0$ is given by Eq. (11), while that at $t>0$ is given by Eqs. (1), (2), (3). Thus at $t=0$ the boson interaction parameter is quenched from $K_{0} \rightarrow K$, and a lattice of strength $g$ is also switched on. Since we are interested in nonequilibrium dynamics, we will use the Keldysh formalism. ${ }^{24} \phi_{-/+}$will denote fields that are (time/anti-time)-ordered on the Keldysh axes. Accordingly we may define Green's functions $G_{a b}$, with $a, b= \pm$ where $G_{--}(12)=-i\left\langle T \phi_{-}(1) \phi_{-}(2)\right\rangle$ is the timeordered Green's function, $G_{++}(12)=-i\left\langle\tilde{T} \phi_{+}(1) \phi_{+}(2)\right\rangle$ is the anti-time ordered Green's function, $G_{-+}(12)=$ $-i\left\langle\phi_{+}(2) \phi_{-}(1)\right\rangle$ and $G_{+-}(12)=-i\left\langle\phi_{+}(1) \phi_{-}(2)\right\rangle$.

It is also convenient to define quantum $\left(\phi_{q}\right)$ and classical fields $\left(\phi_{c l}\right)$,

$$
\phi_{ \pm}=\frac{1}{\sqrt{2}}\left(\phi_{c l} \mp \phi_{q}\right)
$$

with respect to which the basic retarded (R), advanced (A) and Keldysh (K) Green's functions are,

$$
\begin{aligned}
G_{R}\left(x t, y t^{\prime}\right) & =-i \theta\left(t-t^{\prime}\right)\left\langle\left[\phi(x t), \phi\left(y t^{\prime}\right)\right]\right. \\
& =-i\left\langle\phi_{c l}(x t) \phi_{q}\left(y t^{\prime}\right)\right\rangle \\
G_{A}\left(x t, y t^{\prime}\right) & =i \theta\left(t^{\prime}-t\right)\left\langle\left[\phi(x t), \phi\left(y t^{\prime}\right)\right]\right. \\
& \left.=-i\left\langle\phi_{q}(x t) \phi_{c l}\left(y t^{\prime}\right)\right\rangle\right\rangle \\
G_{K}\left(x t, y t^{\prime}\right) & =-i\left\langle\left\{\phi(x t), \phi\left(y t^{\prime}\right)\right\}\right\rangle \\
& =-i\left\langle\phi_{c l}(x t) \phi_{c l}\left(y t^{\prime}\right)\right\rangle
\end{aligned}
$$

Note that $G_{R, A, K}$ are linear combinations of $G_{a b}$. In what follows we will use lower case letters $g_{R, A, K}, g_{a b}$ to denote the Green's functions for the free theory, and upper-case for the Green's functions in the presence of the periodic potential.

The Green's function in the presence of a periodic potential is modified as follows

$$
\begin{aligned}
& i G_{c d}(1,2)=i g_{c d}(1,2)-\frac{1}{2}\left(\frac{g u}{\alpha^{2}}\right)^{2} \sum_{a, b= \pm} \epsilon^{a b} \int d 3 \int d 4 \\
& \left\langle\cos \left(\gamma \phi_{a}(3)\right) \cos \left(\gamma \phi_{b}(4)\right) \phi_{c}(1) \phi_{d}(2)\right\rangle+\ldots
\end{aligned}
$$

where 1,2,3,4 denote both position and time indices, $\epsilon^{++}=\epsilon^{--}=1$ and $\epsilon^{+-}=\epsilon^{-+}=-1$, while \langle\rangle are expectation values evaluated in the initial state which is the ground state of the free theory with interaction parameter $K_{0}$. Note that the fact that the periodic potential was switched on suddenly at $t=0$ only appears in the lower limit of the time integration. In general any switching protocol may be employed.

Performing the contractions, one finds,

$$
\begin{aligned}
& \left\langle\cos \left(\gamma \phi_{a}(3)\right) \cos \left(\gamma \phi_{b}(4)\right) \phi_{c}(1) \phi_{d}(2)\right\rangle \\
& =\left\langle\cos \left(\gamma \phi_{a}\right) \cos \left(\gamma \phi_{b}\right)\right\rangle\left(i g_{c d}+\gamma^{2}\left[g_{c a} g_{a d}+g_{c b} g_{b d}\right.\right. \\
& \left.\left.-g_{c a} g_{b d}-g_{c b} g_{a d}\right]\right)
\end{aligned}
$$



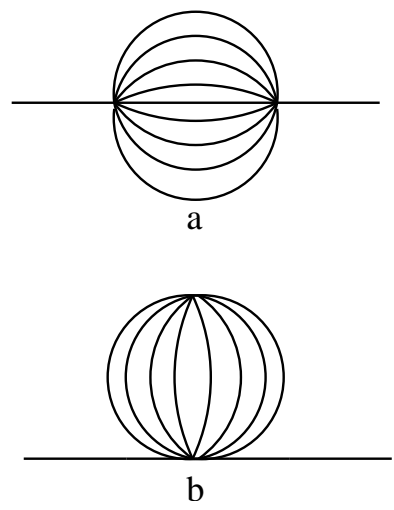

FIG. 2. The self-energies to leading order $\mathcal{O}\left(g^{2}\right)$ in the periodic potential. The solid lines are the boson Green's function G.

While the first disconnected term cancels when summing on indices $a, b$, the remaining terms may be used to identify a self-energy $\Sigma$ to $\mathcal{O}\left(g^{2}\right)$ which is shown diagrammatically in Fig. 2, and whose formal expression is,

$$
\begin{array}{r}
\Sigma^{R}(1,2)=\Pi^{R}(1,2)-\delta(1-2) \int d 3 \Pi^{R}(1,3) \\
\Sigma^{K}(1,2)=\Pi^{K}(1,2)
\end{array}
$$

where,

$$
\begin{aligned}
& \Pi^{R}\left(x_{1} t_{1}, x_{2} t_{2}\right)=-i \gamma^{2}\left(\frac{g u}{\alpha^{2}}\right)^{2} \theta\left(t_{1}-t_{2}\right) \\
& {\left[\left\langle\cos \left(\gamma \phi_{-}\left(x_{1} t_{1}\right)\right) \cos \left(\gamma \phi_{-}\left(x_{2} t_{2}\right)\right)\right\rangle\right.} \\
& \left.-\left\langle\cos \left(\gamma \phi_{+}\left(x_{1} t_{1}\right)\right) \cos \left(\gamma \phi_{+}\left(x_{2} t_{2}\right)\right)\right\rangle\right] \\
& \Pi^{K}\left(x_{1} t_{1}, x_{2} t_{2}\right)=-i \gamma^{2}\left(\frac{g u}{\alpha^{2}}\right)^{2} \\
& {\left[\left\langle\cos \left(\gamma \phi_{-}\left(x_{1} t_{1}\right)\right) \cos \left(\gamma \phi_{-}\left(x_{2} t_{2}\right)\right)\right\rangle\right.} \\
& \left.+\left\langle\cos \left(\gamma \phi_{+}\left(x_{1} t_{1}\right)\right) \cos \left(\gamma \phi_{+}\left(x_{2} t_{2}\right)\right)\right\rangle\right]
\end{aligned}
$$

While the effect of this self-energy has been studied in the past in order to understand equilibrium finite temperature properties ${ }^{54}-56$ of $1 \mathrm{D}$ systems, here we will be interested on its effect on dynamics arising after a quench.

The Dyson equation obtained from the infinite summation of terms $G=g+g \Sigma g+g \Sigma g \Sigma g+\ldots$ generalized to the time-dependent problem, may now be written as,

$$
\begin{aligned}
& G_{R}\left(x t, y t^{\prime}\right)=g_{R}\left(x t, y t^{\prime}\right)+\int d x_{1} d t_{1} \int d x_{2} d t_{2} \\
& \times g_{R}\left(x t, x_{1} t_{1}\right) \Pi^{R}\left(x_{1} t_{1}, x_{2} t_{2}\right)\left[G_{R}\left(x_{2} t_{2}, y t^{\prime}\right)\right. \\
& \left.-G_{R}\left(x_{1} t_{1}, y t^{\prime}\right)\right] \\
& =g_{R}\left(x t, y t^{\prime}\right)+\int d x_{1} d t_{1} \int d x_{2} d t_{2} \\
& \times g_{R}\left(x t, x_{1} t_{1}\right) \Sigma^{R}\left(x_{1} t_{1}, x_{2} t_{2}\right) G_{R}\left(x_{2} t_{2}, y t^{\prime}\right) \\
& G_{K}\left(x t, y t^{\prime}\right)=\int d x_{1} d t_{1} \int d x_{2} d t_{2} G_{R}\left(x t, x_{1} t_{1}\right) \\
& \times \Pi^{K}\left(x_{1} t_{1}, x_{2} t_{2}\right) G_{A}\left(x_{2} t_{2}, y t^{\prime}\right)
\end{aligned}
$$

where the retarded propagator for the Luttinger model is

$$
\begin{aligned}
& g_{R}^{-1}(1,2)= \\
& -\frac{1}{\pi K u}\left[\partial_{t_{1}}^{2}-u^{2} \partial_{x_{1}}^{2}\right] \delta\left(t_{1}-t_{2}\right) \delta\left(x_{1}-x_{2}\right)
\end{aligned}
$$

Since the Keldysh component $G_{K}$ contains information on the mode-occupation, its equation of motion constitutes a (quantum) kinetic equation. An alternative way to write the Keldysh component is by expressing $G_{K}$ in the following way,

$$
G_{K} \equiv G_{R} \circ F-F \circ G_{A}
$$

where the symbol $\circ$ implies convolution on $x$ and $t$ and also matrix multiplication. Using the Dyson equation in matrix form $\left(\hat{g}^{-1}-\hat{\Sigma}\right) \circ \hat{G}=\hat{1}$ we obtain the following kinetic equation in terms of the auxiliary function $F$,

$$
F \circ g_{A}^{-1}-g_{R}^{-1} \circ F=\Sigma_{K}-\Sigma_{R} \circ F+F \circ \Sigma_{A}
$$

Assuming spatial invariance which allows us to transform to momentum space, and using that the left hand side of Eq. (42) is $\frac{1}{\pi K u}\left[\partial_{t}^{2}-\partial_{t^{\prime}}^{2}\right] F\left(q, t, t^{\prime}\right)$, and changing variables from $\left(t, t^{\prime}\right)$ to $(T, \tau)=\left(\left(t+t^{\prime}\right) / 2, t-t^{\prime}\right)$, Eq. (42) becomes

$$
\begin{aligned}
\partial_{\tau} \partial_{T} F(q, T, \tau) & =\left(\frac{\pi K u}{2}\right)\left[\Sigma^{K}(q, T, \tau)\right. \\
& \left.-\left(\Sigma^{R} \circ F\right)(q, T, \tau)+\left(F \circ \Sigma^{A}\right)(q, T, \tau)\right](43)
\end{aligned}
$$

So far no approximations have been made other than in the precise diagrams that make up $\Sigma$.

We now define,

$$
\tilde{F}(q, T, \omega)=\int_{-\infty}^{\infty} d \tau \int_{-\infty}^{\infty} d r e^{-i q r+i \omega \tau} F(r, T, \tau)
$$

and employ a gradient expansion to lowest order i.e., $\left(\widetilde{\Sigma^{R} \circ F}\right)(q, T, \omega) \approx \tilde{\Sigma}^{R}(q, T, \omega) \tilde{F}(q, T, \omega) .58$ Note that by dropping the derivatives in the gradient expansion we are assuming that the dynamics is valid for $t$ sufficiently large since for small $t, F$ usually rapidly oscillates $\underline{59}$ This decoupling simplifies the kinetic equation to,

$$
\partial_{T} \tilde{F}(q, T, \omega)=\left(\frac{i \pi K u}{2 \omega}\right)\left[\tilde{\Sigma}^{K}(q, T, \omega)\right.
$$




$$
\left.-\left(\tilde{\Sigma}^{R}(q, T, \omega)-\tilde{\Sigma}^{A}(q, T, \omega)\right) \tilde{F}(q, T, \omega)\right]
$$

Let us consider the analogous equation for the spectral density

$$
G_{\rho}=G_{A}-G_{R}
$$

The Dyson equations for the retarded and advanced propagators are,

$$
\begin{aligned}
& \left(g_{R}^{-1} \circ G_{R}\right)\left(x x_{0}, y y_{0}\right)=1+\int d z \int_{y_{0}}^{x_{0}} d z_{0} \\
& \times \Sigma^{R}\left(x x_{0}, z z_{0}\right) G_{R}\left(z z_{0}, y y_{0}\right) \\
& \left(g_{A}^{-1} \circ G_{A}\right)\left(x x_{0}, y y_{0}\right)=1+\int d z \int_{x_{0}}^{y_{0}} d z_{0} \\
& \times \Sigma^{A}\left(x x_{0}, z z_{0}\right) G_{A}\left(z z_{0}, y y_{0}\right)
\end{aligned}
$$

where $x, y, z$ denote spatial coordinates and $x_{0}, y_{0}, z_{0}$ denote temporal coordinates. Taking the difference of the above two equations and noting that terms such as $\theta\left(t_{1}-t_{1}^{\prime}\right) \int_{t_{1}^{\prime}}^{t_{1}} d t_{2} \Sigma^{R}(1,2) G^{A}\left(2,1^{\prime}\right)=0$ we obtain,

$$
\begin{aligned}
& \left(g^{-1} \circ\right) G_{\rho}\left(x x_{0}, y y_{0}\right)=\int d z \int_{y_{0}}^{x_{0}} d z_{0} \\
& \times\left[\Sigma^{R}\left(x x_{0}, z z_{0}\right)-\Sigma^{A}\left(x x_{0}, z z_{0}\right)\right] G_{\rho}\left(z z_{0}, y y_{0}\right)
\end{aligned}
$$

By applying the $g^{-1}$ operator on the second argument, and combining the result with the above equation, we obtain

$$
\begin{aligned}
& {\left[g^{-1} \circ G_{\rho}-G_{\rho} \circ g^{-1}\right]\left(x x_{0}, y y_{0}\right)=\int d z \int_{y_{0}}^{x_{0}} d z_{0}} \\
& \times\left[\Sigma^{R}\left(x x_{0}, z z_{0}\right)-\Sigma^{A}\left(x x_{0}, z z_{0}\right)\right] G_{\rho}\left(z z_{0}, y y_{0}\right) \\
& -G_{\rho}\left(x x_{0}, z z_{0}\right)\left[\Sigma^{R}\left(z z_{0}, y y_{0}\right)-\Sigma^{A}\left(z z_{0}, y y_{0}\right)\right]
\end{aligned}
$$

As before we now go into center of mass and relative coordinates, and assuming that the system is spatially homogeneous, we find that to leading order in the gradient expansion,

$$
\omega \partial_{T} \tilde{G}_{\rho}(q, T, \omega)=0
$$

In other words, the lowest order gradient expansion captures the dynamics of the mode occupation and neglects the time evolution of the spectral density, which is fixed by the state before the quench. From the definition (41) we note that $F$ comes multiplied by $G_{\rho}$ which is a sharply peaked function,

$$
G_{\rho}(q, \omega)=\frac{i \pi^{2} K}{|q|}[\delta(\omega-u|q|)-\delta(\omega+u|q|)]
$$

The kinetic equation can therefore be simplified by being computed only at the on-shell frequencies $\omega= \pm u q$ (below we simplify the notation by replacing $\tilde{\Sigma}, \tilde{F}$ by $\Sigma, F)$,

$$
\begin{aligned}
& \partial_{T} F(q, T, \omega=u q)=\left(\frac{i \pi K}{2 q}\right)\left[\Sigma^{K}(q, T, \omega=u q)\right. \\
& \left.-\left(\Sigma^{R}-\Sigma^{A}\right)(q, T, \omega=u q) F(q, T, \omega=u q)\right]
\end{aligned}
$$

Thus the leading order gradient expansion is identical to the so called "quasi-classical" approximation which assumes that the spectral density remains sharply peaked.

There is a hidden assumption in going into the mixed representation of $\omega, T$. The time evolution of the system begins at $t=0$ but in order to be able to Fourier transform we need to extend the range of the relative coordinate $\tau$ from $-\infty$ to $\infty$ in (44). Therefore by extending $t=0$ to $t=-\infty$ we are using a hybrid description, since the initial conditions for the kinetic equation are defined at a finite time $t=0$. This approximation implies that the kinetic-equation cannot be used to describe dynamics at times too short after the quench at $t=0$, but works best at slightly longer times where some of the memory effects have decayed away. A detailed comparison of the kinetic equation with the full solution of the Dyson equation for $\phi^{4}$ theory and other models like the Yukawa model may be found in Refs. 25, 28, 59, and 60. Such a comparison for our model with the cosine potential is beyond the scope of this paper.

The self-energies entering in the kinetic equation under the on-shell approximation for the spectral density are,

$$
\begin{aligned}
& \Sigma^{K}(q, T, \omega)=-i\left(\frac{g u \gamma}{\alpha^{2}}\right)^{2} \int_{-\infty}^{\infty} d \tau \int_{-\infty}^{\infty} d r \cos (q r) \cos (\omega \tau) \\
& e^{-I(T, r, \tau)} \cos \left[K_{\mathrm{eq}} \sum_{\varepsilon= \pm 1} \tan ^{-1}\left(\frac{u \tau+\varepsilon r}{\alpha}\right)\right] \\
& \left(\Sigma^{R}-\Sigma^{A}\right)(q, T, \omega) \\
& =-i\left(\frac{g u \gamma}{\alpha^{2}}\right)^{2} \int_{-\infty}^{\infty} d \tau \int_{-\infty}^{\infty} d r \sin (\omega \tau) \cos (q r) \\
& e^{-I(T, r, \tau)} \sin \left[K_{\mathrm{eq}} \sum_{\varepsilon= \pm 1} \tan ^{-1}\left(\frac{u \tau+\varepsilon r}{\alpha}\right)\right]
\end{aligned}
$$

where,

$$
\begin{array}{r}
I(T, r, \tau)=K_{\text {eq }} \sum_{\varepsilon= \pm 1} \int_{0}^{\infty} \frac{d q}{q} e^{-\alpha q} F(u q, T) \\
{[1-\cos (q(u \tau+\varepsilon r))]}
\end{array}
$$

In what follows we will suppress the frequency label as it is understood that it is fixed at an on-shell value, and use only the arguments $q$ and the time $T$ to label quantities. The initial condition for the boson distribution function follows from the discussion in Section $\amalg \mathrm{A}$ and is given by

$$
F(q, T=0)=1+2\left\langle\gamma_{q}^{\dagger} \gamma_{q}\right\rangle(T=0)=\frac{K_{\text {neq }}}{K_{\text {eq }}}
$$

In the absence of a quench, the distribution function is given by the zero temperature limit of $\operatorname{coth}\left(\frac{u|q|}{2 T_{\mathrm{eq}}}\right) \rightarrow 1$. Due to the interaction quench, a highly nonequilibrium boson distribution Eq. (57) is generated. In the absence of the cosine potential this distribution function is infinitely long lived. However the cosine potential allows for 
inelastic multi-particle scattering, which will relax it. We will study how this relaxation comes about and whether the system thermalizes, and if so on what time-scales.

Eqs. (53), (54), (55), (56) together with the initial condition in Eq. (57) define the problem we wish to solve. Note that the equilibrium distribution function $F=\operatorname{coth} \frac{u|q|}{2 T_{e q}}$ is a solution of the kinetic equation for any $T_{\text {eq }}$, as it should be. Due to the self-energies being of the form $\Sigma \sim\langle\cos (\gamma \phi) \cos (\gamma \phi)\rangle \sim e^{-\gamma^{2}\langle\phi \phi\rangle}$, where $\langle\phi \phi\rangle$ is proportional to the boson distribution function, the matrix elements determining the in-scattering and outscattering rates in the kinetic equation (53) have an exponential dependence on the distribution function through the function $e^{-I} \sim e^{-F}$. This is in marked contrast to $\phi^{4}$ theory or other fermionic models where the matrix elements entering the kinetic equation have a polynomial dependence on the distribution function, for example for two-particle scattering, the matrix elements are proportional to $F F(1 \pm F)(1 \pm F)$. Hence we use the term multi-particle scattering to describe the effect of the cosine potential.

It is instructive to study the form of the self-energy $\Sigma(r, 0) \sim\langle\cos (\gamma \phi)(r) \cos (\gamma \phi)(0)\rangle$ at zero temperature

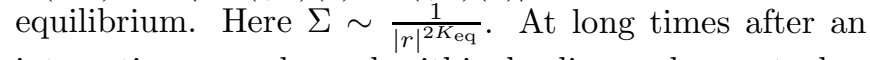
interaction quench, and within leading order perturbation theory where $F$ may be taken to be the distribution function right after the quench (Eq. (57)), $\Sigma \sim \frac{1}{|r|^{2} K_{\text {neq }}}$. Since $K_{\text {neq }}>K_{\text {eq }}$, the matrix element for scattering is suppressed by the interaction quench.

The above exponential dependence of the matrix elements on the distribution function is a consequence of the orthogonality catastrophe arising due to the interaction quench. In particular the larger the quench amplitude $K_{\text {neq }}-K_{\text {eq }}$, the poorer is the overlap between the initial wave-function and the low-energy eigenstates of the final Hamiltonian, leading to an exponential suppression of the matrix elements entering the kinetic equation. Because of our bosonization approach, this physics is captured naturally. This also shows that a quench, however small in magnitude, cannot lead to a suitable linearization procedure for the kinetic equation as is possible to do for small deviations about thermal equilibrium 39

It is important to now summarize the conservation laws associated with Eq. (53). The potential $\cos (\gamma \phi)$ does not conserve particle number, this is also the case for $\phi^{4}$ theory ${ }^{25}$ However since our system is closed, energy is conserved. The total energy has a kinetic contribution, and an interaction contribution arising from the cosine potential. In the next section we carefully evaluate the conserved energy and prove that the kinetic equation (53) obtained from the leading order gradient expansion is a conserving approximation. Readers not interested in the derivation of the conserved energy may go straight to the results in Section $\mathrm{V}$.

\section{2PI FORMALISM AND ENERGY CONSERVATION}

If we perform standard perturbative approximations for the self-energies (36) and (37), such as replacing the dressed Green's function in the self-energy in Fig. 2. by the bare one, we violate energy conservation. $\underline{\underline{61}}$ An efficient scheme to approach this problem and to develop conserving approximations is via the two-particle irreducible (2PI) effective action. $30,62,63$ The 2PI effective action involves a diagrammatic series in terms of closed loops where dressed propagators are used instead of bare ones $\underline{64}$ By applying a variational principle on the $2 \mathrm{PI}$ functional, the exact Dyson equations (38) and (39) are obtained. The approximations are performed directly on the effective action by truncating the corresponding diagrammatic expansion. By construction, the effective action (and consequently it's truncations) is invariant under global transformations of the Green's function $G$, and using Noether's theorem we can find expressions for the conserved quantities (in our specific case the energy) $\stackrel{27}{?}$ The 2PI effective action $\Gamma(G)$ is given by,

$$
\begin{aligned}
& \Gamma(G) \\
& =\frac{i}{2} \int d x d t \int d x^{\prime} d t^{\prime} \ln \left(G^{-1}\right)\left(x t, x^{\prime} t^{\prime}\right) \delta\left(x-x^{\prime}\right) \delta\left(t-t^{\prime}\right) \\
& +\frac{i}{2} \int d x d t \int d x^{\prime} d t^{\prime} g^{-1}\left(x t, x^{\prime} t^{\prime}\right) G\left(x^{\prime} t^{\prime}, x t\right)+\Gamma_{2}(G)
\end{aligned}
$$

where $G\left(x, t ; x^{\prime}, t^{\prime}\right)=\left\langle T_{C} \varphi(x, t) \varphi\left(x^{\prime}, t^{\prime}\right)\right\rangle$ is the Schwinger-Keldysh contour-ordered Green's function and $i g^{-1}\left(x t, x^{\prime} t^{\prime}\right)=-\left(\partial_{t}^{2}-\partial_{x}^{2}\right) \delta\left(x-x^{\prime}\right) \delta\left(t-t^{\prime}\right)$ is the free propagator (where we have set $u=1, \pi K=1$ ). Since we are interested in finding the energy-momentum tensor $T^{\mu \nu}$ we apply a general (space and time dependent) infinitesimal translation,

$$
\begin{aligned}
x & \rightarrow x+\varepsilon_{1}(x, t) \\
t & \rightarrow t+\varepsilon_{0}(x, t)
\end{aligned}
$$

which vanishes at the boundaries. To leading order in $\varepsilon(x, t)$ the corresponding transformation of $G\left(x t, x^{\prime} t^{\prime}\right)$ is

$$
\begin{aligned}
& \delta G\left(x t, x^{\prime} t^{\prime}\right) \\
& =\frac{\partial G\left(x t, x^{\prime} t^{\prime}\right)}{\partial x} \varepsilon_{1}(x, t)+\frac{\partial G\left(x t, x^{\prime} t^{\prime}\right)}{\partial t} \varepsilon_{0}(x, t) \\
& +\frac{\partial G\left(x t, x^{\prime} t^{\prime}\right)}{\partial x^{\prime}} \varepsilon_{1}\left(x^{\prime}, t^{\prime}\right)+\frac{\partial G\left(x t, x^{\prime} t^{\prime}\right)}{\partial t^{\prime}} \varepsilon_{0}\left(x^{\prime}, t^{\prime}\right) \\
& =\left[\varepsilon_{\mu}(x, t) \partial_{\mu}+\varepsilon_{\mu}\left(x^{\prime}, t^{\prime}\right) \partial_{\mu^{\prime}}\right] G\left(x t, x^{\prime} t^{\prime}\right)
\end{aligned}
$$

Above we used the notation $x_{\mu}=(x, t), x_{\mu^{\prime}}=\left(x^{\prime}, t^{\prime}\right)$, and a sum over repeated indices is implied.

We will show below that we can write the variation of $\Gamma(G)$ as,

$$
\delta \Gamma(G)=\int d x \int d t T^{\mu \nu}(x, t) \tilde{\partial}_{\mu} \varepsilon_{\nu}(x, t)
$$

The above equation defines the energy-momentum tensor $T^{\mu \nu}$. The $\tilde{\partial}_{\mu}$ is defined as $\tilde{\partial}_{\mu}=\left(\tilde{\partial}_{x}, \tilde{\partial}_{t}\right) \equiv\left(-\partial_{x}, \partial_{t}\right)$. 
After an integration by parts this becomes,

$$
\delta \Gamma(G)=-\int d x d t \tilde{\partial}_{\mu} T^{\mu \nu}(x, t) \varepsilon_{\nu}(x, t)
$$

Now, the Dyson equation is derived from the effective action $\Gamma(G)$ by requiring that $\frac{\delta \Gamma}{\delta G}=0$. By taking the functional derivative of $\Gamma(G)$ we obtain,

$$
\frac{\delta \Gamma}{\delta G}=-\frac{i}{2} G^{-1}\left(x t, x^{\prime} t^{\prime}\right)+\frac{i}{2} g^{-1}\left(x t, x^{\prime} t^{\prime}\right)+\frac{\delta \Gamma_{2}}{\delta G}
$$

Setting this to zero we obtain the Dyson equation,

$$
G^{-1}\left(x, t ; x^{\prime}, t^{\prime}\right)-g^{-1}\left(x, t ; x^{\prime}, t^{\prime}\right)+2 i \frac{\delta \Gamma_{2}}{\delta G}=0
$$

provided that

$$
\Sigma\left(x, t ; x^{\prime}, t^{\prime}\right)=2 i \frac{\delta \Gamma_{2}}{\delta G}
$$

Thus we have,

$$
G^{-1}\left(x t, x^{\prime} t^{\prime}\right)=g^{-1}\left(x t, x^{\prime} t^{\prime}\right)-\Sigma\left(x t, x^{\prime} t^{\prime}\right)
$$

Now we see that if $G$ satisfies the Dyson equation, Eqs. (61) and (62) are zero. Moreover, since $\left(\varepsilon_{0}, \varepsilon_{1}\right)$ is arbitrary, the energy-momentum tensor is conserved

$$
\tilde{\partial}_{\mu} T^{\mu \nu}=0
$$

The above implies

$$
\partial_{t} T^{00}-\partial_{x} T^{x 0}=0
$$

Integrating over all space and noting that the second term above is a total derivative and therefore vanishes, leads to the definition of the conserved energy $E=$ $\int d x T^{00}$ such that,

$$
\frac{d E}{d t}=\frac{d}{d t} \int_{\text {all space }} d x T^{00}(x, t)=0
$$

For a spatially homogeneous system like the one we study, the energy density $T^{00}$ is position independent and is also conserved. In this section we will derive an explicit expression for $T^{00}$.

We can obtain the explicit expression for $T^{\mu \nu}$ by varying (58). We first define $\delta \Gamma(G)=\delta \Gamma_{a}(G)+\delta \Gamma_{b}(G)+$ $\delta \Gamma_{2}(G)$ corresponding to varying the first, second and third term in Eq. (58). We first vary $\Gamma_{a}(G)$

$$
\delta \Gamma_{a}(G)=-\frac{i}{2} \delta \operatorname{Tr}(\ln G)=-\frac{i}{2} \operatorname{Tr} G^{-1} \delta G
$$

This can be written explicitly as,

$$
\begin{aligned}
\left.-\frac{i}{2} \int_{\left[\varepsilon_{\mu}(x, t)\right.} d x d t \partial_{\mu} G\left(x^{\prime} t^{\prime}, x t\right)+\varepsilon_{\mu}\left(x^{\prime}, t^{\prime}\right) \partial_{\mu^{\prime}} G\left(x^{\prime} t^{\prime}, x t\right)\right]
\end{aligned}
$$

Introducing a $\delta$-function in the dummy variables $\left(x_{2}, t_{2}\right)$, we can rewrite the first term as (the second term is analogous),

$$
-\frac{i}{2} \int d x d t \int d x^{\prime} d t^{\prime} \int d x_{2} d t_{2} \delta\left(x-x_{2}\right) \delta\left(t-t_{2}\right) \varepsilon_{\mu}\left(x_{2}, t_{2}\right)
$$

$$
\begin{aligned}
& G^{-1}\left(x t, x^{\prime} t^{\prime}\right) \partial_{2 \mu} G\left(x^{\prime} t^{\prime}, x_{2} t_{2}\right) \\
&=-\frac{i}{2} \int d x d t \int d x_{2} d t_{2} \delta\left(x-x_{2}\right) \delta\left(t-t_{2}\right) \varepsilon_{\mu}\left(x_{2}, t_{2}\right) \partial_{2 \mu} \int d x^{\prime} d t^{\prime} G^{-1}\left(x t, x^{\prime} t^{\prime}\right) G\left(x^{\prime} t^{\prime}, x_{2} t_{2}\right) \\
&=- \frac{i}{2} \int d x d t \int d x_{2} d t_{2} \delta\left(x-x_{2}\right) \delta\left(t-t_{2}\right) \varepsilon_{\mu}\left(x_{2}, t_{2}\right) \\
&=-\frac{i}{2} \int d x_{2} d t_{2} \varepsilon_{\mu}\left(x_{2}, t_{2}\right) \partial_{2 \mu}[\delta(0) \delta(0)]=0
\end{aligned}
$$

Treating the second term in a similar way we find $\delta \Gamma_{a}(G)=0$ and therefore does not contribute anything to the stress tensor.

The expression for $\delta \Gamma_{b}(G)$ is,

$$
\begin{aligned}
& \delta \Gamma_{b}(G)=\frac{1}{2} \int d x d t \int d x^{\prime} d t^{\prime} \delta\left(x-x^{\prime}\right) \delta\left(t-t^{\prime}\right)\left(\partial_{x}^{2}-\partial_{t}^{2}\right) \\
& {\left[\varepsilon_{1}(x, t) \partial_{x} G\left(x^{\prime} t^{\prime}, x t\right)+\varepsilon_{1}\left(x^{\prime}, t^{\prime}\right) \partial_{x^{\prime}} G\left(x^{\prime} t^{\prime}, x t\right)\right.} \\
& \left.\quad+\varepsilon_{0}(x, t) \partial_{t} G\left(x^{\prime} t^{\prime}, x t\right)+\varepsilon_{0}\left(x^{\prime}, t^{\prime}\right) \partial_{t^{\prime}} G\left(x^{\prime} t^{\prime}, x t\right)\right]
\end{aligned}
$$

The above may be written in a short-hand form,

$$
\begin{aligned}
& \delta \Gamma_{b}(G) \\
= & \frac{1}{2} \int d x d t \int d x^{\prime} d t^{\prime} \delta\left(x-x^{\prime}\right) \delta\left(t-t^{\prime}\right) \\
& \left(\partial_{x}^{2}-\partial_{t}^{2}\right)\left[\varepsilon_{\mu}(x, t) \partial_{\mu} G\left(x^{\prime} t^{\prime}, x t\right)+\varepsilon_{\mu}\left(x^{\prime}, t^{\prime}\right) \partial_{\mu^{\prime}} G\left(x^{\prime} t^{\prime}, x t\right)\right]
\end{aligned}
$$

If we now use the following expression

$$
\partial_{a}^{2}\left(\varepsilon_{\mu} \partial_{\mu} G\right) \stackrel{O(\varepsilon)}{=} \varepsilon_{\mu} \partial_{a}^{2}\left(\partial_{\mu} G\right)+2\left(\partial_{a} \varepsilon_{\mu}\right) \partial_{a}\left(\partial_{\mu} G\right)
$$

where $\partial_{a}^{2}$ is $\partial_{x}^{2}$ or $\partial_{t}^{2}$ and perform a few manipulations we get,

$$
\begin{aligned}
& \delta \Gamma_{b}(G) \\
& =\int_{\left[\partial_{t} \varepsilon_{\mu}(x, t)\right.} d x d t \int d x^{\prime} d t^{\prime} \delta\left(x-x_{t}-\partial_{x} \varepsilon_{\mu}(x, t) \delta\left(t-t^{\prime}\right)\right. \\
& \left.\quad-\frac{1}{2} \partial_{\mu} \varepsilon_{\mu}(x, t)\left(\partial_{t t^{\prime}}^{2}-\partial_{x x^{\prime}}^{2}\right)\right] G\left(x^{\prime} t^{\prime}, x t\right)
\end{aligned}
$$

The above may be rewritten as (defining $\tilde{\delta}_{\mu \nu}=$ $\left.\left(-\delta_{x x}, \delta_{t t}\right)\right)$

$$
\begin{aligned}
& \delta \Gamma_{b}(G)=\int d x d t \int d x^{\prime} d t^{\prime} \delta\left(x-x^{\prime}\right) \delta\left(t-t^{\prime}\right) \\
& {\left[\left(\tilde{\partial}_{\nu} \epsilon_{\mu}\right) \partial_{\mu^{\prime}} \partial_{\nu}-\frac{1}{2}\left(\tilde{\partial}_{\nu} \epsilon_{\mu}\right) \tilde{\delta}_{\mu \nu}\left(\partial_{t t^{\prime}}^{2}-\partial_{x x^{\prime}}^{2}\right)\right] G\left(x^{\prime} t^{\prime}, x t\right)}
\end{aligned}
$$

which has the required form (61). We thus find the following contribution to the energy-momentum tensor

$$
\begin{aligned}
& T_{\text {kin }}^{\mu \nu}(x, t)=\int d x^{\prime} d t^{\prime} \delta\left(x-x^{\prime}\right) \delta\left(t-t^{\prime}\right) \\
& \quad \times\left[\frac{\partial^{2} G\left(x^{\prime}, t^{\prime} ; x, t\right)}{\partial x_{\mu} \partial x^{\prime}{ }_{\nu}}-\frac{1}{2} \tilde{\delta}_{\mu \nu} \frac{\partial^{2} G\left(x^{\prime}, t^{\prime} ; x, t\right)}{\partial x_{\lambda} \tilde{\partial} x^{\prime}{ }_{\lambda}}\right]
\end{aligned}
$$


The label kin is to indicate that this is the contribution to the tensor for free bosons. In the presence of interactions, even though we will continue to use the label kin, $T_{\text {kin }}^{\mu \nu}$ contains contributions from the interactions as the Green's function $G$ is affected by the interactions. However in the presence of interactions, an additional contribution to the tensor arises from varying the last term in Eq. (58), which we discuss shortly.

Since the kinetic equation is expressed in terms of $F$ or equivalently $G_{K}$, it is necessary to express the contour ordered Green's function $G$ in terms of $G_{K}$ and $G_{\rho}$. Defining $\theta_{C}\left(t-t^{\prime}\right)$ to be the step function along the Keldysh contour, the decomposition identity is given by, 25

$$
\begin{aligned}
& i G\left(x, t ; x^{\prime}, t^{\prime}\right)=i\left\langle\phi(x, t) \phi\left(x^{\prime}, t^{\prime}\right)\right\rangle \theta_{C}\left(t-t^{\prime}\right) \\
& +i\left\langle\phi\left(x^{\prime}, t^{\prime}\right) \phi(x, t)\right\rangle \theta_{C}\left(t^{\prime}-t\right) \\
& =\frac{1}{2} i \overbrace{\left\langle\left\{\phi(x, t), \phi\left(x^{\prime}, t^{\prime}\right)\right\}\right\rangle}^{-G_{K}\left(x, t ; x^{\prime}, t^{\prime}\right)}+ \\
& =\frac{1}{2} \overbrace{\left\langle\left\langle\left[\phi(x, t), \phi\left(x^{\prime}, t^{\prime}\right)\right]\right\rangle\right.}^{G_{\rho}\left(x, t ; x^{\prime}, t^{\prime}\right)} \overbrace{\operatorname{sgn}_{C}\left(t-t^{\prime}\right)}^{\theta_{C}\left(t-t^{\prime}\right)-\theta_{C}\left(t^{\prime}-t\right)} \\
& =-\frac{1}{2}\left[G_{K}\left(x, t ; x^{\prime}, t^{\prime}\right)-G_{\rho}\left(x, t ; x^{\prime}, t^{\prime}\right) \operatorname{sgn}_{C}\left(t-t^{\prime}\right)\right]
\end{aligned}
$$

and for the self-energy since $G^{-1}=g^{-1}-\Sigma$,

$$
\begin{aligned}
& i \Sigma\left(x, t ; x^{\prime}, t^{\prime}\right) \\
& =\frac{1}{2}\left[\Sigma^{K}\left(x, t ; x^{\prime}, t^{\prime}\right)-\Sigma^{\rho}\left(x, t ; x^{\prime}, t^{\prime}\right) \operatorname{sgn}_{\mathrm{C}}\left(t-t^{\prime}\right)\right]
\end{aligned}
$$

Then we can write $T_{\text {kin }}^{\mu \nu}(x, t)$ in terms of $G_{K}$ only since

$$
G(x, t ; x, t)=\frac{i}{2} G_{K}(x, t ; x, t) .
$$

Equation (77) becomes

$$
\begin{aligned}
& T_{\mathrm{kin}}^{\mu \nu}(x, t)=\int d x^{\prime} d t^{\prime} \delta\left(x-x^{\prime}\right) \delta\left(t-t^{\prime}\right) \\
& {\left[\frac{i}{2} \frac{\partial^{2} G_{K}\left(x^{\prime}, t^{\prime} ; x, t\right)}{\partial x_{\mu} \partial x^{\prime}{ }_{\nu}}-\frac{i}{4} \tilde{\delta}_{\mu \nu} \frac{\partial^{2} G_{K}\left(x^{\prime}, t^{\prime} ; x, t\right)}{\partial x_{\lambda} \tilde{\partial} x^{\prime}{ }_{\lambda}}\right]}
\end{aligned}
$$

Thus the kinetic part of the energy density $T_{\mathrm{kin}}^{00}(x, t)$ after restoring the Luttinger liquid parameters reads,

$$
\begin{gathered}
T_{\mathrm{kin}}^{00}(x, t)=\frac{1}{\pi K u} \int d x^{\prime} d t^{\prime} \delta\left(x-x^{\prime}\right) \delta\left(t-t^{\prime}\right) \\
{\left[\frac{i}{4} \frac{\partial^{2} G_{K}\left(x^{\prime}, t^{\prime} ; x, t\right)}{\partial t \partial t^{\prime}}+\frac{i}{4} u^{2} \frac{\partial^{2} G_{K}\left(x^{\prime}, t^{\prime} ; x, t\right)}{\partial x \partial x^{\prime}}\right]}
\end{gathered}
$$

In consonance with the kinetic equation (53) we perform a gradient expansion to lowest order, which as we showed before is equivalent to the on-shell or quasiclassical approximation. This along with the assumption that the system is homogeneous gives (defining

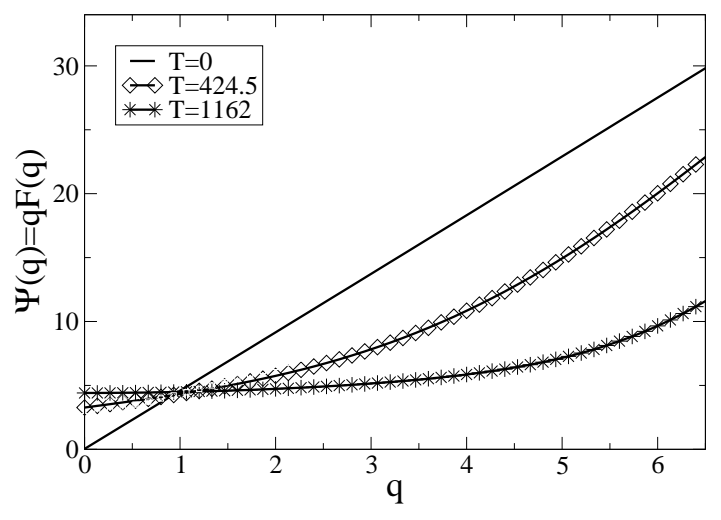

FIG. 3. The distribution function $\Psi(q)=q F(q)$ plotted for increasing times for a quench where $K_{\text {eq }}=3, K_{\text {neq }}=13.8$. At the initial time, the quench generates the distribution given by the straight line $\Psi(q)=q \frac{K_{\text {neq }}}{K_{\text {eq }}}$. The distribution converges to the equilibrium distribution given by $\Psi(q)=q \operatorname{coth}\left(\frac{u q}{2 T_{\mathrm{eq}}}\right)$.

$$
\begin{aligned}
T & \left.=\left(t+t^{\prime}\right) / 2, \tau=t-t^{\prime}\right) \\
& T_{\mathrm{kin}}^{00}(T)= \\
& \frac{i}{(4 \pi K u)} \int_{-\infty}^{\infty} \frac{d q}{2 \pi} \int d \tau \delta(\tau) \\
& \times\left(\frac{1}{4} \partial_{T}^{2}-\partial_{\tau}^{2}+u^{2} q^{2}\right) G_{K}(q, T, \tau) \\
& \approx \frac{i}{(4 \pi K u)} \int_{-\infty}^{\infty} \frac{d q}{2 \pi} \int_{-\infty}^{\infty} \frac{d \omega}{2 \pi}\left(\omega^{2}+u^{2} q^{2}\right) G_{K}(q, T, \omega)
\end{aligned}
$$

where a term $O\left(\partial_{T}^{2}\right)$ was dropped. Using

$$
\begin{aligned}
& G_{K}(q, \omega, T)= \\
& -\frac{i \pi^{2} K}{|q|}[\delta(\omega-u|q|)-\delta(\omega+u|q|)] F(q, \omega, T)
\end{aligned}
$$

we obtain (suppressing the $\omega$ label as it is understood that it is evaluated at $\pm u q$ )

$$
T_{\mathrm{kin}}^{00}(T)=\frac{u}{4 \pi} \int_{-\infty}^{\infty} d q e^{-\alpha|q|}|q| F(q, T)
$$

This is the expected expression for the kinetic energy. In the absence of the cosine potential, this quantity is exact, and was evaluated in Section II.

Let us now construct the stress-momentum tensor from the third term in Eq. (58), and from that construct the interaction contribution to the conserved energy. The contribution from the last term $\Gamma_{2}$ can be written as,

$$
\delta \Gamma_{2}(G)=\int d x d t \int d x^{\prime} d t^{\prime} \overbrace{\frac{\delta \Gamma_{2}(G)}{\delta G\left(x t, x^{\prime} t^{\prime}\right)}}^{(1 / 2 i) \Sigma\left(x^{\prime} t^{\prime}, x t\right)}
$$




$$
\begin{aligned}
& \times\left[\varepsilon_{\mu}(x, t) \partial_{\mu} G\left(x t ; x^{\prime} t^{\prime}\right)+\varepsilon_{\mu}\left(x^{\prime}, t^{\prime}\right) \partial_{\mu^{\prime}} G\left(x t, x^{\prime} t^{\prime}\right)\right] \\
& =\frac{1}{2 i} \int d x d t \int d x^{\prime} d t^{\prime} \Sigma\left(x^{\prime}, t^{\prime} ; x, t\right) \\
& \times\left[\varepsilon_{\mu}(x, t) \partial_{\mu} G\left(x t ; x^{\prime} t^{\prime}\right)+\varepsilon_{\mu}\left(x^{\prime}, t^{\prime}\right) \partial_{\mu^{\prime}} G\left(x t, x^{\prime} t^{\prime}\right)\right](86)
\end{aligned}
$$

Changing variables in the second term we obtain an expression of the form (62),

$$
\delta \Gamma_{2}(G)=\frac{1}{2 i} \int_{\left.\Sigma\left(x t ; x^{\prime} t^{\prime}\right) \partial_{\mu} G\left(x^{\prime} t^{\prime}, x t\right)\right] \varepsilon_{\mu}(x, t)} d x d t x^{\prime} d t^{\prime}\left[\Sigma\left(x^{\prime} t^{\prime}, x t\right) \partial_{\mu} G\left(x t ; x^{\prime} t^{\prime}\right)+\right.
$$

where we identify

$$
\begin{aligned}
\tilde{\partial}_{\mu} T_{\Sigma}^{\mu \nu}(x, t)= & -\frac{1}{2 i} \int d x^{\prime} d t^{\prime}\left[\Sigma\left(x^{\prime} t^{\prime}, x t\right) \partial_{\nu} G\left(x t, x^{\prime} t^{\prime}\right)+\right. \\
& \left.\Sigma\left(x t, x^{\prime} t^{\prime}\right) \partial_{\nu} G\left(x^{\prime} t^{\prime}, x t\right)\right]
\end{aligned}
$$

It is convenient to write the above expression in terms of $G_{\rho}$ and $G_{K}$ (see Appendix $\mathrm{A}$ for intermediate steps) to obtain,

$$
\begin{aligned}
\tilde{\partial}_{\mu} T_{\Sigma}^{\mu \nu}(x, t)= & \frac{1}{2 i} \int d x^{\prime} \int_{0}^{t} d t^{\prime}\left[-\Sigma^{\rho}\left(x^{\prime} t^{\prime}, x t\right) \partial_{\nu} G_{K}\left(x t, x^{\prime} t^{\prime}\right)\right. \\
& \left.+\Sigma^{K}\left(x^{\prime} t^{\prime}, x t\right) \partial_{\nu} G_{\rho}\left(x t, x^{\prime} t^{\prime}\right)\right]
\end{aligned}
$$

Integrating over $x$, the left hand side of the above equation is

$$
\int d x \tilde{\partial}_{\mu} T_{\Sigma}^{\mu 0}(x, t)=\int d x \partial_{t} T_{\Sigma}^{00}(x, t) \overbrace{\int d x \partial_{x} T_{\Sigma}^{00}(x, t \gamma(90)}^{\text {surface terms } \rightarrow 0}
$$

Assuming spatial invariance, the expression for the interaction contribution to the conserved energy density $T_{\Sigma}^{00}(t)$ is found to be,

$$
\begin{aligned}
& T_{\Sigma}^{00}(t)=\frac{1}{2 i} \int_{0}^{t} d t^{\prime \prime} \int_{0}^{t^{\prime \prime}} d t^{\prime} \int_{-\infty}^{\infty} \frac{d q}{2 \pi} e^{-\alpha|q|} \\
& {\left[\Sigma^{K}\left(q, t^{\prime}, t^{\prime \prime}\right) \partial_{t^{\prime \prime}} G_{\rho}\left(q, t^{\prime \prime}, t^{\prime}\right)\right.} \\
& \left.-\Sigma^{\rho}\left(q, t^{\prime}, t^{\prime \prime}\right) \partial_{t^{\prime \prime}} G_{K}\left(q, t^{\prime \prime}, t^{\prime}\right)\right]
\end{aligned}
$$

The next step is to perform a lowest order gradient expansion as we did before for the kinetic equation and for $T_{\text {kin }}^{\mu \nu}$. It is more convenient to work with $d T_{\Sigma}^{00}(t) / d t$ and then integrate over $t$. The expression for $d T_{\Sigma}^{00}(t) / d t$ is,

$$
\begin{aligned}
& \frac{d T_{\Sigma}^{00}(t)}{d t}=\frac{1}{2 i} \int_{0}^{t} d t^{\prime} \int_{-\infty}^{\infty} \frac{d q}{2 \pi} e^{-\alpha|q|} \\
& {\left[\Sigma^{K}\left(q, t^{\prime}, t\right) \partial_{t} G_{\rho}\left(q, t, t^{\prime}\right)\right.} \\
& \left.-\Sigma^{\rho}\left(q, t^{\prime}, t\right) \partial_{t} G_{K}\left(q, t, t^{\prime}\right)\right]
\end{aligned}
$$

Rewriting this expression using Wigner coordinates

$$
\begin{aligned}
& (T, \tau)=\left(\frac{t+t^{\prime}}{2}, t-t^{\prime}\right) \text { one obtains, } \\
& \frac{d T_{\Sigma}^{00}(t)}{d t}=\frac{1}{2 i} \int_{-\infty}^{\infty} \frac{d q}{2 \pi} \int_{0}^{t} d t^{\prime} e^{-\alpha|q|} \\
& \quad\left[\Sigma^{K}\left(q, \frac{\left(t+t^{\prime}\right)}{2}, t^{\prime}-t\right) \partial_{t} G_{\rho}\left(q, \frac{\left(t+t^{\prime}\right)}{2}, t-t^{\prime}\right)\right. \\
& \quad-(\rho \leftrightarrow K)],
\end{aligned}
$$

The next step is to Fourier transform with respect to the relative coordinate $\tau=t-t^{\prime}$. As mentioned before, in order to go to $\omega$-space we must extend the interval of integration of the relative coordinate $\tau$ from $(0, t)$ to $(-\infty, \infty)$. Noticing that $\int_{0}^{\infty} d \tau e^{i\left(\omega-\omega^{\prime}\right) \tau} \rightarrow \pi \delta\left(\omega-\omega^{\prime}\right)$, using $\partial_{t} G_{\rho}\left(q, t, t^{\prime}\right)=-i \int \frac{d \omega}{2 \pi} e^{-i \omega\left(t-t^{\prime}\right)} \omega \tilde{G}_{\rho}(q, T, \omega)$ and finally integrating over $T$ we obtain the expression for $T_{\Sigma}^{00}(T)$

$$
\begin{gathered}
T_{\Sigma}^{00}(T)=-\frac{1}{4} \int_{0}^{T} d T^{\prime} \int_{-\infty}^{\infty} \frac{d q}{2 \pi} e^{-\alpha|q|} \int \frac{d \omega}{2 \pi} \omega\left[\tilde{\Sigma}^{K}\left(q, T^{\prime}, \omega\right)\right. \\
\left.\times \tilde{G}^{\rho}(q, \omega)-(\rho \leftrightarrow K)\right]
\end{gathered}
$$

The final step is to use the on-shell approximation which as shown before follows from the leading order gradient expansion,

$$
\begin{aligned}
& T_{\Sigma}^{00}(T)=-\frac{1}{4} \int_{0}^{T} d T^{\prime} \int_{-\infty}^{\infty} \frac{d q}{2 \pi} e^{-\alpha|q|} \int \frac{d \omega}{2 \pi} \omega \\
& {\left[\tilde{\Sigma}^{K}\left(q, T^{\prime}, \omega\right) \tilde{G}_{\rho}(q, \omega)\right.} \\
& \left.+\tilde{\Sigma}^{\rho}\left(q, T^{\prime}, \omega\right) \tilde{G}_{\rho}(q, \omega) F\left(q, T^{\prime}, \omega\right)\right] \\
& =-\frac{i \pi^{2} K}{16 \pi^{2}} \int_{0}^{T} d T^{\prime} \int_{-\infty}^{\infty} d q e^{-\alpha|q|} \int d \omega \frac{\omega}{|q|}\left[\tilde{\Sigma}^{K}\left(q, T^{\prime}, \omega\right)+\right. \\
& \left.\tilde{\Sigma}^{\rho}\left(q, T^{\prime}, \omega\right) F\left(q, T^{\prime}, \omega\right)\right][\delta(\omega-u|q|)-\delta(\omega+u|q|)] \\
& =-\frac{i K u}{8} \int_{0}^{T} d T^{\prime} \int_{-\infty}^{\infty} d q e^{-\alpha|q|\left[\tilde{\Sigma}^{K}\left(q, T^{\prime}\right)\right.} \\
& \left.+\tilde{\Sigma}^{\rho}\left(q, T^{\prime}\right) F\left(q, T^{\prime}\right)\right]
\end{aligned}
$$

In summary the conserved energy density is

$$
\frac{E}{L}=T_{\mathrm{kin}}^{00}(T)+T_{\Sigma}^{00}(T)
$$

with $T_{\text {kin }}^{00}$ defined in Eq. (85) and $T_{\Sigma}^{00}$ given by,

$$
\begin{aligned}
& T_{\Sigma}^{00}(T)=-\frac{i K u}{8} \int_{0}^{T} d T^{\prime} \int_{-\infty}^{\infty} d q e^{-\alpha|q|}\left[\Sigma^{K}\left(q, T^{\prime}\right)\right. \\
& \left.+\Sigma^{\rho}\left(q, T^{\prime}\right) F\left(q, T^{\prime}\right)\right]
\end{aligned}
$$


On differentiating Eq. (96) in time and using the kinetic equation (53), we see that the total energy is conserved by the kinetic equation since,

$$
\begin{aligned}
& \frac{1}{L} \frac{d E(T)}{d T}=\frac{i u K}{4} \int_{0}^{\infty} d q e^{-\alpha q}\left[\Sigma^{K}(q, T)+\Sigma^{\rho}(q, T) F(q, T)\right] \\
& -\frac{i u K}{4} \int_{0}^{\infty} d q e^{-\alpha q}\left[\Sigma^{K}(q, T)+\Sigma^{\rho}(q, T) F(q, T)\right] \\
& =0
\end{aligned}
$$

Thus the kinetic equation derived by us is a conserving approximation, with the conserved energy given in Eq. (96).

\section{RESULTS}

In this section we numerically solve the kinetic equation (53) with the in-scattering and out-scattering rates given in Eqs. (54), (55), (56), and the boundary condition that at $T=0$ the distribution function is given by Eq. (57). As discussed in the previous sections, the cosine potential does not conserve particle number, but the quench always conserves the total energy. Using the 2PI formalism we found the expression for the conserved energy to be Eq. (96). It is the sum of a kinetic part Eq. (85) and an interaction part Eq. (97). We find it convenient to label the energies this way even though the interactions affect the kinetic part of the energy as well because they affect the single-particle Green's function. In case the system thermalizes, we would like to understand what is the equilibrium temperature at which it should thermalize. This temperature has a kinetic contribution as well as a correction from the cosine interaction.

We find that after the leading order gradient expansion there is another zero mode in the problem, namely

$$
\frac{d T_{\mathrm{kin}}^{00}(T)}{d T}=0 ; \frac{d T_{\Sigma}^{00}(T)}{d T}=0
$$

The proof of this is given in Appendix B. The above implies that under the leading order gradient expansion, the total kinetic energy (summing over all momentum modes) and the total potential energy are separately conserved. This additional conservation law implies that since at the initial time $T_{\Sigma}^{00}(T=0)=0$, it remains zero always. This implies that the equilibrium temperature at which the system thermalizes can be calculated in a rather straightforward way using the analysis of Section I A for the quadratic Luttinger model. In particular the equilibrium temperature $T_{\text {eq }}$ even in the presence of the cosine potential is given by Eq. (25) within the leading order gradient expansion.

The appearance of this zero mode where the total kinetic energy of the bosons is conserved is not an accident. Recall that even for $\phi^{4}$ theories or interacting fermionic models, a kinetic equation obtained from a leading order gradient and quasi-particle approximation results in two and three particle scattering processes where the total kinetic energy of the particles is conserved. The interactions at most give a Hartree correction to the kinetic energy. While our kinetic equation is more complicated than that for two and three particle scattering, yet the leading order gradient expansion leads to the same result.

All our numerical computations are done for $g=0.1$ and $\gamma=2$. Moreover we set the velocity $u=1$, and all energy and length scales are in units of the cut-off $\Lambda=u / \alpha$. It is also interesting to note that the strength of $g$ only appears as an over-all multiplying factor in the kinetic equation (53) so that as $g$ decreases, the timeevolution slows proportionally as $g^{2}$.

It is convenient to define the function

$$
\Psi(q)=q F(q)
$$

Since in equilibrium $\Psi(q)=q \operatorname{coth} \frac{u q}{2 T_{\mathrm{eq}}}$, the $q=0$ intercept of $\Psi(q)$ may be used to define an effective temperature,

$$
T_{\mathrm{eff}}=\frac{u}{2} \Psi(q=0)
$$

Fig. 3 shows how the distribution function evolves in time. At $T=0$ the interaction quench generates a nonequilibrium distribution function $\Psi(q, T=0)=$ $q \frac{K_{\text {neq }}}{K_{\text {eq }}}$. The cosine potential leads to inelastic scattering that relaxes this distribution function and generates an effective temperature $T_{\text {eff }}$ which corresponds to a nonzero intercept at $q=0$ in Fig. 3. Eventually at long times this effective temperature evolves into the true thermal temperature $T_{\text {eq. }}$. Fig. 4 shows the distribution function at sufficiently long times for two different quenches, and compares it with the equilibrium distribution function. Not only the intercept at $q=0$, but also the functional form of the distribution function agrees very well with the equilibrium form. There is some deviation at large q, but we expect that this deviation will become smaller at longer times. To determine the long distance behavior of various correlation functions, it is ultimately the distribution function at small $q$ that is important. Thus for all practical purposes, Fig. 4 is a fully thermalized distribution function.

\section{A. Toy model to recapture the dynamics for large quenches}

Simulating the full kinetic equation is numerically costly. Fig. 3] shows that the distribution function stays mostly smooth as a function of $q$, and ultimately it is the small $q$ behavior which is most important in determining the in-scattering and out-scattering rates. This observation allows us to simulate the entire time-evolution using a toy model where we approximate the distribution function by its value in the vicinity of $q=0$,

$$
\Psi(q>0, T)=2 \frac{T_{\mathrm{eff}}(T)}{u}+B(T) q+A(T) q^{2}+C(T) q^{3}
$$




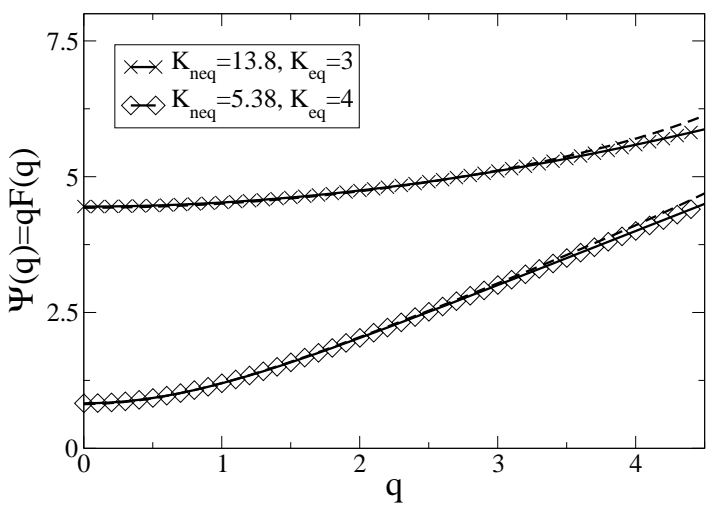

FIG. 4. At long times the distribution converges to $\Psi_{e q}(q)=$ $q \operatorname{coth}\left(\frac{u q}{2 T_{\mathrm{eq}}}\right)$ (dashed line). The quenches and the time for which the distributions are shown correspond to $K_{\text {eq }}=$ $3, K_{\text {neq }}=13.8, T=1162$ and $K_{\text {eq }}=4, K_{\text {neq }}=5.38, T=$ 1072. The final temperatures are respectively $T_{\text {eq }}=2.22$ and $T_{\text {eq }}=0.414$.

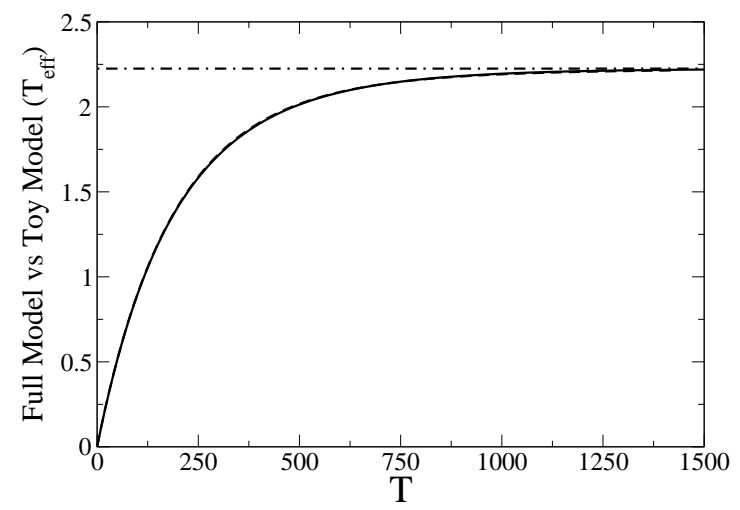

FIG. 5. Comparison between the full model (dashed line) and the toy model (solid line) for $K_{\text {eq }}=3$ and $K_{\text {neq }}=13.8$. The differences are unobservable. The system thermalizes to $T_{\text {eq }}$ (dot-dashed line).

where the coefficients $T_{\text {eff }}, B, A, C$ are respectively the intercept, slope, and curvatures of the distribution function at $q=0$. From inversion symmetry $\Psi(q)=\Psi(-q)$. Substituting in Eq. (53), the full kinetic equation may be reduced to a set of coupled rate equations describing how these four coefficients evolve in time, where the boundary conditions are

$$
\begin{aligned}
& A(T=0)=C(T=0)=T_{\text {eff }}(T=0)=0 ; \\
& B(T=0)=\frac{K_{\text {neq }}}{K_{\text {eq }}}
\end{aligned}
$$

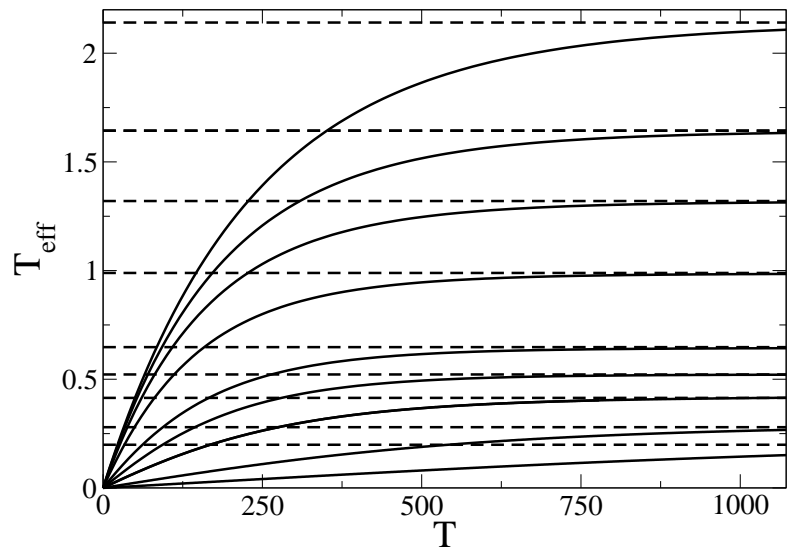

FIG. 6. Time-evolution of the effective temperature for (bottom to top) $K_{\text {neq }}=4.40,4.72,5.38,6.0,6.72,9.08,11.48,13.91$ and 17.72 with $K_{\text {eq }}=4$. For all quenches the system thermalizes to the equilibrium temperate $T_{\text {eq }}$ (dashed line).

The rate equations are found to be,

$$
\begin{aligned}
& \frac{d T_{\mathrm{eff}}(T)}{d T}=\frac{\pi K}{2}\left[\frac{u}{2} i \Sigma^{K}(q=0, T)\right. \\
& \left.-i\left(\Sigma^{R}-\Sigma^{A}\right)(q=0, T) T_{\mathrm{eff}}(T)\right] \\
& \frac{d B(T)}{d T}=-\frac{\pi K}{2} i\left(\Sigma^{R}-\Sigma^{A}\right)(q=0, T) B(T) \\
& \frac{d A(T)}{d T}=\frac{\pi K}{2}\left[-i\left(\Sigma^{R}-\Sigma^{A}\right)(q=0, T) A(T)\right. \\
& \left.+\frac{1}{2}\left(\left.\frac{\partial^{2} i \Sigma^{K}}{\partial q^{2}}\right|_{q=0}-\left.\frac{2 T_{\mathrm{eff}}(T)}{u} \frac{\partial^{2} i\left(\Sigma^{R}-\Sigma^{A}\right)}{\partial q^{2}}\right|_{q=0}\right)\right]
\end{aligned}
$$

where the coefficient $C$ is determined by imposing energy conservation and in particular $\frac{d T_{\Sigma}^{00}}{d T}=0$. The selfenergies and their momentum derivatives that appear above depend on the coefficients $T_{\text {eff }}, A, B, C$ through their dependence on the distribution function entering in the exponent $I$ in Eqs. (54) and (55). A comparison between the full solution of the kinetic equation (53) and the above toy model is shown in Fig. [5 where the timeevolution of the effective temperature after a quench is plotted. The two agree very well. For smaller quench amplitudes however the deviation between the toy model and the full kinetic equation becomes larger because for smaller quenches the dynamics, at least for short times, is affected by distribution function at all $q$.

Fig. [6 shows how the effective temperature evolves in time after a quench where $K_{\text {eq }}=4$ while $K_{\text {neq }}$ is varied from 4.4 to 17.72 . The larger quenches $\left(K_{\text {neq }} \geq 6.0\right)$ are obtained from the toy model, while the smaller quenches are obtained from solving the full kinetic equation. For all cases, the system is found to thermalize at the equilibrium temperature $T_{\text {eq }}$ shown as a dashed line. 


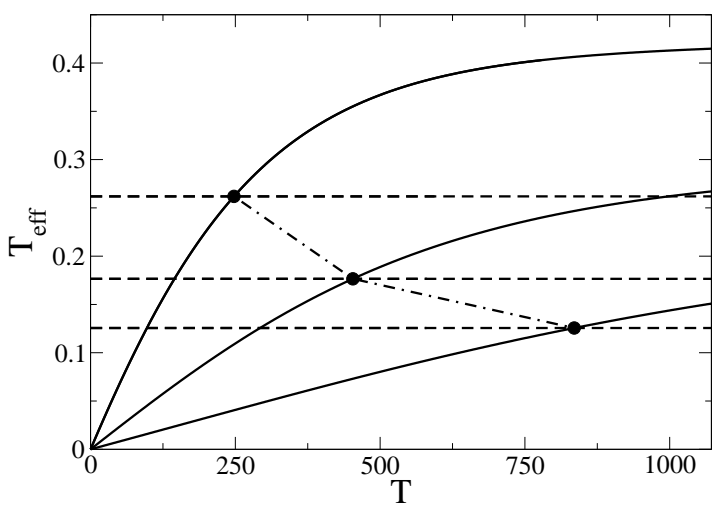

FIG. 7. For small quenches, the relaxation times (given by the abscissa corresponding to the three black dots) decreases with increasing quench amplitude (dot-dashed line). The parameters are $K_{\text {eq }}=4$ and $K_{\text {neq }}=4.40,4.72$ and 5.38 (bottom to top).

\section{B. Time-scales for thermalization}

An important question concerns the time-scales for thermalization. Assuming that the relaxation is purely exponential $T_{\text {eff }}(T)=T_{\text {eq }}\left[1-e^{-\eta T}\right]$, we define the thermalization time $\left(T_{\mathrm{th}}=1 / \eta\right)$ as the time at which the effective temperature is related to the equilibrium temperature as follows,

$$
T_{\text {eff }}\left(T_{\mathrm{th}}=1 / \eta\right)=T_{\text {eq }}\left[1-e^{-1}\right]
$$

We find an interesting non-monotonic dependence of the thermalization time $1 / \eta$ on the quench amplitude $K_{\text {neq }}-K_{\text {eq }}$. Naively one expects the thermalization time to increase as the quench amplitude decreases, approaching infinity for zero quench amplitude $K_{\text {eq }}=K_{\text {neq }}$. Note that a sudden switching on of $g$, keeping the interaction parameter fixed also generates nonequilibrium dynamics, but this cannot be captured within the leading order gradient expansion.

In contrast to the above expectation we find that the thermalization time increases with decreasing quench amplitude (or the thermalization rate increases with increasing quench amplitude) only for small quenches around equilibrium $K_{\text {neq }}-K_{\text {eq }} \lesssim K_{\text {eq. }}$. For large quenches on the other hand $K_{\text {neq }}-K_{\text {eq }} \gtrsim K_{\text {eq }}$, this behavior is reversed. Fig. 7 shows the expected increase in thermalization time for decreasing quench amplitudes for small quenches, while Fig. 8 shows how this behavior is reversed for larger quenches.

A summary of the relaxation rates (inversely related to the thermalization time) for quenches with $K_{\text {eq }}=3$ and $K_{\text {eq }}=4$ is shown in Fig. 9, and clearly shows the nonmonotonic behavior with a maximum relaxation rate for some optimal $K_{\text {neq }}$ for each $K_{\text {eq }}$. The non-monotonic behavior arises due to the dependence of the thermalization time on two competing effects. One is the dependence of the scattering rate on the available phase-space for scattering that increases with increasing quench amplitude

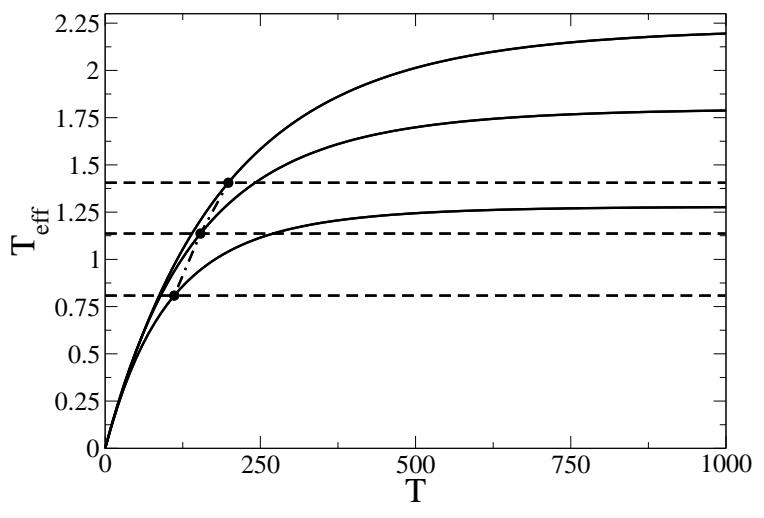

FIG. 8. For large quenches, the relaxation time (given by the abscissa corresponding to the three black dots) increases with the quench amplitude (dot-dashed line). The parameters are $K_{\text {eq }}=3$ and $K_{\text {neq }}=8.39,11.3$ and 13.8 (bottom to top).

and therefore favors an increase in the thermalization rate with increasing quench amplitude. The second effect is that of the orthogonality catastrophe where a quench results in an initial state which has only a partial overlap with the low-energy eigenstates of the Hamiltonian after the quench, with the wavefunction overlap becoming poorer with increasing quench amplitude. This behavior is reflected by the exponential dependence of the matrix elements entering the kinetic equation on the distribution function $F$. Thus as the quench amplitude grows, $F$ increases, suppressing the scattering rates, and ultimately leading to a non-monotonic dependence of the thermalization time on the quench amplitude.

It is interesting to ask how much of the numerical results may be recovered from a perturbative estimate for the inelastic scattering rate. We use Eq. (53) to define an inelastic scattering rate,

$$
\eta_{0}=\lim _{\omega \rightarrow 0}\left(\frac{\pi K}{2}\right) \frac{i}{\omega}\left(\Sigma^{R}-\Sigma^{A}\right)(q, T, \omega=u q)
$$

where $\Sigma^{R, A}$ depends on $F$ which is determined using the kinetic equation. Such an inelastic scattering rate occurs in equilibrium and finite temperature as well for weak deviations of the distribution function from thermal equilibrium, and has been used to determine the finite-temperature lifetimes of the sound modes of the Luttinger liquid 56 We discuss the finite-temperature expression in Appendix C.

For our problem, we may make a crude estimate for the inelastic scattering rate by using perturbation theory, where the distribution function $F$ entering in Eq. (108) is taken to be the value right after the quench, $F=\frac{K_{\text {neq }}}{K_{\text {eq }}}$. This is equivalent to replacing the dressed Green's function in the self-energy by its bare value. While this is not a conserving approximation, it still gives a result which shares many features with the results obtained from the kinetic equation. As shown in Appendix C, the pertur- 


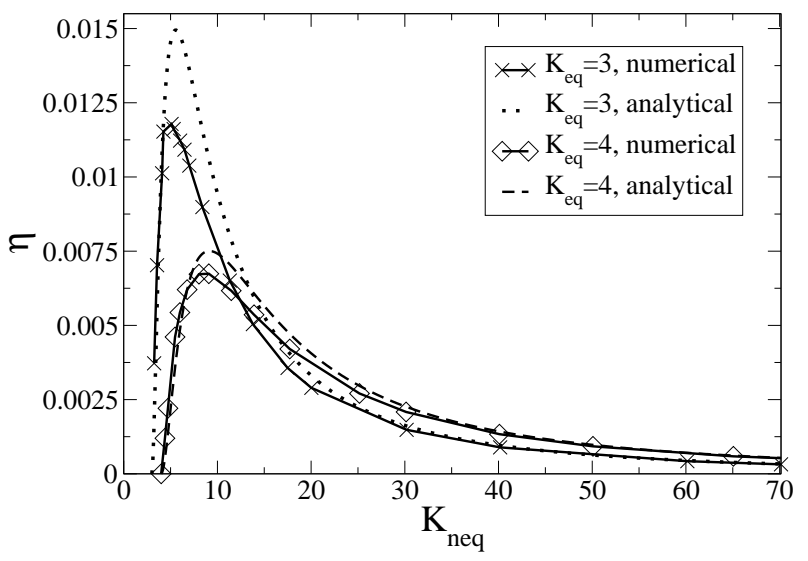

FIG. 9. The relaxation rate (inverse thermalization time) for quenches with different $K_{\text {neq }}$ keeping $K_{\text {eq }}$ fixed at $K_{\text {eq }}=3$ and $K_{\text {eq }}=4$. $\left[K_{\text {neq }}-K_{\text {eq }}\right] / K_{\text {eq }}$ is proportional to the energy density injected due to the quench. The numerical results are compared with the analytic expression Eq. (109) obtained from perturbation theory.

bative estimate for $\eta_{0}$ in units of the cut-off is $\underline{42,65}$

$$
\begin{aligned}
& \eta_{0}=\left(\frac{\pi K}{2}\right) g^{2} \gamma^{2} \\
& \times\left[\frac{\pi}{2^{K_{\text {neq }}-1}\left(K_{\text {neq }}-1\right) B\left(\frac{K_{\text {eq }}+K_{\text {neq }}}{2}, \frac{K_{\text {neq }}-K_{\mathrm{eq}}}{2}\right)}\right] \\
& \times\left[\frac{\pi}{2^{K_{\text {neq }}-2}\left(K_{\text {neq }}-2\right) B\left(\frac{K_{\text {eq }}+K_{\text {neq }}-2}{2}, \frac{K_{\text {neq }}-K_{\text {eq }}}{2}\right)}\right) \\
& \left.-\frac{\pi}{2^{K_{\text {neq }}-2}\left(K_{\text {neq }}-2\right) B\left(\frac{K_{\mathrm{eq}}+K_{\text {neq }}}{2}, \frac{K_{\text {neq }}-K_{\mathrm{eq}}-2}{2}\right)}\right]
\end{aligned}
$$

Fig. 9 shows a comparison between the rate obtained from the kinetic equation, and Eq. (109). The agreement is impressive, especially for small and large quenches. The optimal value of $K_{\text {neq }}$ at which the rate is maximum is also in very good agreement. For intermediate quench amplitudes, a slight suppression of the actual relaxation rate compared with the perturbative estimate is found.

It is also interesting to ask how the thermalization time behaves as one approaches the critical point $K_{\text {eq }}=2$. Fig. 9 shows that the thermalization rates for quenches in the vicinity of $K_{\mathrm{eq}}=3$ is overall greater than the thermalization rates in the vicinity of $K_{\text {eq }}=4$. Thus our results indicate that as one approaches the critical point, the system relaxes faster. This observation has also been made in other theoretical and experimental studies,, ,66,67

It is instructive to see how the analytic expression in Eq. (109) behaves for small and large quench amplitudes.
For small quenches $K_{\text {neq }}-K_{\text {eq }} \ll 1$,

$$
\begin{aligned}
& \eta_{0}\left(K_{\text {neq }}-K_{\text {eq }} \ll 1\right) \simeq \\
& \frac{\pi^{3} K}{2} g^{2} \gamma^{2} \frac{2^{-2 K_{\text {eq }}} K_{\text {eq }}}{\left(K_{\text {eq }}-2\right)\left(K_{\text {eq }}-1\right)^{2}}\left(K_{\text {neq }}-K_{\text {eq }}\right)^{2}
\end{aligned}
$$

Thus according to perturbation theory the thermalization rate diverges as one approaches the critical point at $K_{\text {eq }}=2$. This divergence is unphysical and an indication that bare perturbation theory does not work in the vicinity of the critical point, and a more self-consistent approach employing a kinetic equation is necessary. In this paper we do not attempt to study the dynamics too close to the critical point using the kinetic equation and leave it for future studies. In the vicinity of the critical point, it may also be necessary to use renormalization group to improve on our perturbative expression for the self-energy because the cosine potential is a marginal perturbation near the critical point.

Avoiding the above complications, away from the critical point, the thermalization rate obtained from perturbation theory is found to behave as $g^{2}\left(K_{\text {neq }}-K_{\text {eq }}\right)^{2} \sim$ $g^{2}\left(K_{0}-K\right)^{4}$ for small quenches, and is therefore proportional to $g^{2} \times$ the square of the energy injected into the system due to the quench. For large quenches $K_{\text {neq }}-K_{\text {eq }} \gg 1$, the thermalization rate is found to decrease with $K_{\text {neq }}$ as

$$
\eta_{0}\left(K_{\text {neq }}-K_{\text {eq }} \gg 1\right) \simeq \pi g^{2} \gamma^{2} \frac{K_{\text {eq }}}{K_{\text {neq }}^{2}}
$$

We now address the question of whether the approach to thermal equilibrium is truly exponential or not. Fig. 10] shows how the actual time-evolution of the effective temperature compares with a purely relaxational model $T_{\text {eff }}^{\exp }(T)=T_{\text {eq }}\left[1-e^{-\eta T}\right]$. For large quenches, the differences between the two are unobservable. However as the quench amplitude is reduced, the time-evolution deviates more and more from a purely exponential relaxation. The thermalization time is not precisely defined when the relaxation is not exponential, and so our definition for the thermalization time in Eq. (107) becomes somewhat ad hoc for small quenches. Yet the close agreement with the analytic result shows that the definition chosen is still a close estimate of the physical time scale.

\section{CONCLUSIONS AND OUTLOOK}

We have presented a detailed study of quench dynamics of a $1 \mathrm{D}$ system of interacting bosons in a commensurate periodic potential. A quantum kinetic equation was derived perturbatively in the strength of the periodic potential and solved within a leading order gradient expansion. Our results are valid for quenches within the gapless (superfluid) phase. The system is found to thermalize at long times. The thermalization time is found to be non-monotonic in the quench amplitude or the amount 


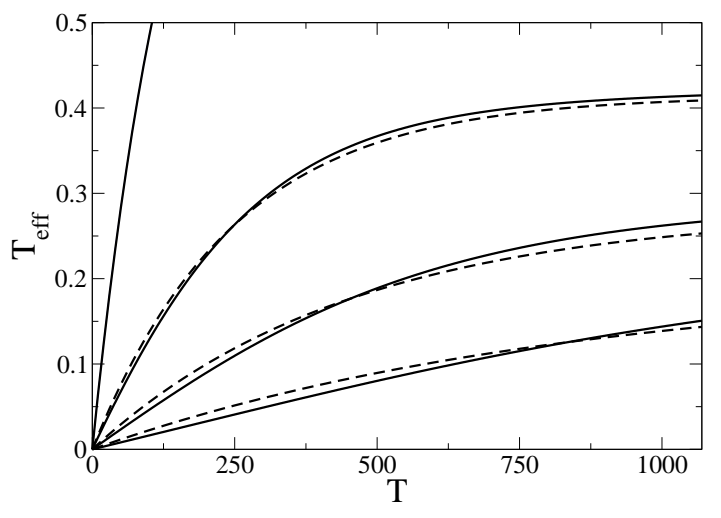

FIG. 10. Comparison between $T_{\text {eff }}(T)$ obtained from the kinetic equation (solid line) with a relaxational ansazt $T_{\mathrm{eff}}^{\exp }(T)=T_{\text {eq }}\left(1-e^{-\eta T}\right)$ (dashed lines). The parameters are $K_{\text {eq }}=4$ and $K_{\text {neq }}=4.4,4.72,5.38$ and 9.08 (bottom to top). As the quench amplitude decreases, the deviation from a simple exponential relaxation becomes larger.

of energy injected into the system. This non-monotonic behavior arises due to a competition between two effects. One is an increase in phase space for scattering as the system is driven further away from equilibrium with increasing quench amplitude, this has the effect of relaxing the system faster as the quench amplitude is increased. The second is an enhancement of the orthogonality catastrophe with increasing quench amplitude arising out of a poorer overlap between the initial wavefunction and the low-energy eigenstates of the final Hamiltonian as the quench amplitude is increased. This has the effect of suppressing the matrix elements for scattering as the quench amplitude is increased, leading to a longer thermalization time. The bosonization approach captures the orthogonality catastrophe naturally. We also find an analytic expression for the relaxation rate from perturbation theory which captures the above features remarkably well.

The result for the non-monotonic dependence of the thermalization time on the quench amplitude can also be understood as follows. Imagine that initially the bosons are in the ground state of a superfluid with interaction parameter $K_{0}$. Now consider increasing the quench amplitude to smaller and smaller values of $K$. Here one would eventually be in the Mott insulator phase where we expect thermalization to be poor or almost absent. A similar behavior is expected when the quench amplitude is increased to larger and larger values of $K$. Here since for $K=\infty$ the bosons are effectively non-interacting, we again expect thermalization to be almost absent for large enough $K$. This non-monotonic behavior of the thermalization time should be observable in experiments if a large tunability of quench amplitudes were possible.

We find that in general the relaxation rates grow as one approaches the critical point. Previous numerical and experimental studies which could approach the critical point both from the superfluid side as well as the Mott insulating side made a similar observation, namely that the relaxation rates are maximal at the critical point and decrease away from it in both directions, $\underline{4,66,67}$

There are many open questions. Firstly we have employed a leading order gradient expansion which works best when the relaxation rates are not too small so that the system looses memory of the initial condition fast. Therefore this approach will not work when $K_{\text {neq }} \rightarrow K_{\text {eq }}$. In particular the leading order gradient expansion gives no dynamics when only the periodic potential is quenched keeping the boson interaction parameter fixed. For this case a full solution of the Dyson equation may be necessary, however this is a numerically challenging task.

The results of this paper are not valid very close to the critical point either. This is because our self-energies were derived perturbatively in the periodic potential, whereas near the critical point, the periodic potential becomes a marginal perturbation. For this case an alternate approach presented in Ref. 68 may be more appropriate where a separation of time-scales was identified. For times smaller than an inelastic scattering rate (but longer than microscopic time-scales), real quantum processes dominate the dynamics and can be treated with a perturbative renormalization group approach, while at longer times the quasi-classical approach of this paper may be employed.

Finally the issue of integrability and the relation of this model to lattice models such as the Bose-Hubbard model and the XXZ chain are open questions. Numerical studies on integrable and non-integrable fermionic models on the lattice show that irrelevant operators do not affect the dynamics for numerically accessible timescales. ${ }^{69}$ Whether there is a fundamental difference between dynamics of lattice models and continuum field theories like the one studied in this paper, or whether this is an issue of differing time-scales is a question that needs to be explored.

Quench dynamics of integrable models described by interacting field theories, and the effect of integrability breaking terms on the dynamics, is an important topic of research. Kinetic equations constructed for some 1D integrable models such as the nearest-neighbor fermionic Hubbard chain,, 36 fermionic models with deltafunction interactions in real space,$^{70}$ the fermionic limit of the Lieb-Liniger model,,$\underset{ }{,}$ and the Calogero-Sutherland model ${ }^{71}$ have been shown to know about the underlying integrability of the systems by either giving vanishing matrix elements for scattering, $, 70,71$ or by causing scattering only in some special points, resulting in nonthermal steady-state solutions. 36 A more systematic study involving other integrable models, and then understanding the effect of integrability breaking terms is an important direction of research.

Acknowledgments: The authors thank I. Aleiner, J. Berges, F. Essler, S. Kehrein and M. Schiró for helpful discussions. This work was partially supported by a grant from the Simons Foundation and by the National Science Foundation under Grants No. PHY 11-25915 and No. DMR-1004589. 
1 I. Bloch, J. Dalibard, and W. Zwerger, Rev. Mod. Phys. 80, 885 (2008).

2 T. Kinoshita, T. Wenger, and D. S. Weiss, Nature (London) 440, 900 (2006).

3 M. Gring, M. Kuhnert, T. Langen, T. Kitagawa, B. Rauer, M. Schreitl, I. Mazets, D. A. Smith, E. Demler, and J. Schmiedmayer, Science 337, 1318 (2012).

4 J. P. Ronzheimer, M. Schreiber, S. Braun, S. S. Hodgman, S. Langer, I. P. McCulloch, F. Heidrich-Meisner, I. Bloch, and U. Schneider, Phys. Rev. Lett. 110, 205301 (2013).

${ }^{5}$ G. Galavotti, The Fermi-Pasta-Ulam Problem: A Status Report (Springer, Berlin, 2008).

6 B. Sutherland, Beautiful Models, World Scientific, Singapore (2004).

7 E. A. Yuzbashyan and B. S. Shastry, J. Stat. Phys. 150, 704 (2013).

8 J.-S. Caux and J. Mossel, J. Stat. Mech. , P02023 (2011).

9 M. Fagotti and F. H. Essler, J. Stat. Mech., P07012 (2013).

10 B. Pozsgay, arXiv:1304.5374 (unpublished).

11 M. Kormos, A. Shashi, Y.-Z. Chou, J.-S. Caux, and A. Imambekov, arXiv:1305.7202 (unpublished).

12 M. Rigol, V. Dunjko, V. Yurovsky, and M. Olshanii, Phys. Rev. Lett. 98, 050405 (2007)

13 D. Fioretto and G. Mussardo, New J. of Phys. 12, 055015 (2010).

14 J.-S. Caux and R. M. Konik, Phys. Rev. Lett. 109, 175301 (2012)

15 G. Mussardo, arXiv:1308.4551, Phys. Rev. Lett. (in print).

16 M. Rigol, Phys. Rev. Lett. 103, 100403 (2009)

17 M. Rigol, Phys. Rev. A 80, 053607 (2009)

18 G. Roux, Phys. Rev. A 79, 021608 (2009)

19 G. Biroli, C. Kollath, and A. M. Läuchli, Phys. Rev. Lett. 105, 250401 (2010).

20 L. F. Santos and M. Rigol, Phys. Rev. E 81, 036206 (2010).

21 J. Mossel and J.-S. Caux, New J. Phys. 14, 075006 (2012).

22 J. M. Deutsch, Phys. Rev. A 43, 2046 (1991).

23 M. Srednicki, Phys. Rev. E 50, 888 (1994)

24 A. Kamenev, Nanophysics: Coherence and Transport, Les Houches 2004 session No. LXXX1 (Elsevier, Amsterdam) (2005).

25 J. Berges, AIP Conf. Proc. 739 , 3 (2004).

26 S. Juchem, W. Cassing, and C. Greiner, Phys. Rev. D 69, 025006 (2004)

27 A. Arrizabalaga, J. Smit, and A. Tranberg, Phys. Rev. D 72, 025014 (2005).

$28 \mathrm{M}$. Lindner and M. M. Müller, Phys. Rev. D 77, 025027 (2008).

29 M. Eckstein, M. Kollar, and P. Werner, Phys. Rev. Lett. 103, 056403 (2009).

30 M. Kronenwett and T. Gasenzer, Applied Physics B 102, 469 (2011).

31 J. Berges, S. Borsányi, and C. Wetterich, Phys. Rev. Lett. 93, 142002 (2004).

32 M. Moeckel $\quad$ and $\quad$ S. Kehrein,

\begin{tabular}{|l|l|}
\hline Phys. Rev. Lett. 100, 175702 (2008) & \\
M. M. Kollar,
\end{tabular}

Phys. Rev. Lett. 100, 120404 (2008)

34 J. Berges, A. Rothkopf, and J. Schmidt,

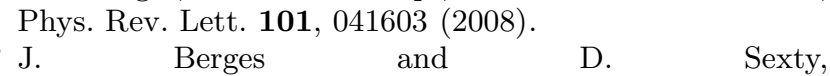

Phys. Rev. Lett. 108, 161601 (2012).

36 M. L. R. Fürst, C. B. Mendl, Phys. Rev. E 86, 031122 (2012).

37 M. L. R. Fürst, C. B. Mendl, Phys. Rev. E 88, 012108 (2013).

38 K. A. Matveev and A. V. Andreev, Phys. Rev. Lett. 107, 056402 (2011)

39 A. Imambekov, T. L. Schmidt, and L. I. Glazman, Rev. Mod. Phys. 84, 1253 (2012)

40 J. Lin, K. A. Matveev, and M. Pustilnik, Phys. Rev. Lett. 110, 016401 (2013).

41 T. Giamarchi, Quantum Physics in One Dimension, Oxford University Press, Oxford (2004).

42 A. Mitra and T. Giamarchi, Phys. Rev. Lett. 107, 150602 (2011)

43 A. Polkovnikov, Annals of Physics 325, 1790 (2010)

44 J. Lancaster, E. Gull, and A. Mitra, Phys. Rev. B 82, 235124 (2010).

45 I. E. Mazets, T. Schumm, and J. Schmiedmayer, Phys. Rev. Lett. 100, 210403 (2008)

46 R. Rajaraman, Solitons and Instantons (North-Holland, Amsterdam, 1982).

47 A. Iucci and M. A. Cazalilla, Phys. Rev. A 80, 063619 (2009)

48 M. A. Cazalilla, Phys. Rev. Lett. 97, 156403 (2006)

49 J. Lancaster and A. Mitra, Phys. Rev. E 81, 061134 (2010).

50 E. Perfetto, Phys. Rev. B 74, 205123 (2006)

51 B. Dóra, M. Haque, and G. Zaránd, Phys. Rev. Lett. 106, 156406 (2011).

52 J Rentrop, D Schuricht and V Meden, New J. of Phys 14, 075001, (2012).

53 M. S. Foster, T. C. Berkelbach, D. R. Reichman, and E. A. Yuzbashyan, Phys. Rev. B 84, 085146 (2011)

${ }^{54}$ H. J. Schulz, Phys. Rev. B 34, 6372 (1986).

55 M. Oshikawa and I. Affleck, Phys. Rev. B 65, 134410 (2002).

56 J. Sirker, R. G. Pereira, and I. Affleck, Phys. Rev. B 83, 035115 (2011).

57 A. Kamenev, Field Theory of Non-Equilibrium Systems, Cambridge University Press, Cambridge (2011).

58 J. Rammer and H. Rev. Mod. Phys. 58, 323 (1986).

59 M. Lindner and M. M. Müller, Phys. Rev. D 73, 125002 (2006).

60 J. Berges and S. Borsányi, Phys. Rev. D 74, 045022 (2006).

61 G. Baym and L. P. Kadanoff, Phys. Rev 124, 287 (1961).

62 Y. B. Ivanov, J. Knoll, and D. N. Voskresensky, Nuclear Physics A 657, 413 (1999).

63 A. Nishiyama, Nuclear Physics A 832, 289 (2010).

64 J. M. Cornwall, R. Jackiw, and E. Tomboulis, Phys. Rev. D 10, 2428 (1974).

65 A. Mitra and T. Giamarchi, Phys. Rev. B 85, 075117 (2012).

66 M. Cramer, A. Flesch, I. P. McCulloch, U. Schollwöck, and J. Eisert, Phys. Rev. Lett. 101, 063001 (2008).

67 S. Trotzky, Y.-A. Chen, A. Flesch, I. McCulloch, U. Schollwöck, J. Eisert, and I. Bloch, Nature Physics 8, 325 (2012).

68 A. Mitra, Phys. Rev. B 87, 205109 (2013).

${ }^{69}$ C. Karrasch, J. Rentrop, D. Schuricht, and V. Meden, 
Phys. Rev. Lett. 109, 126406 (2012).

70 Anders Mathias Lunde, Karsten Flensberg and Leonid I. Glazman, Phys. Rev. B 75, 245418 (2007).

${ }^{71}$ M. Khodas, M. Pustilnik, A. Kamenev, and L. I. Glazman, Phys. Rev. B 76, 155402 (2007).

\section{Appendix A: Derivation of Eq. (89)}

Consider a Keldysh contour $\left(\int_{C}\right)$ starting from an initial time $t_{0}$, going upto a maximum time $t_{\max }$ and then returning to the initial time $t_{0}$. Then the following identities hold 25

$$
\begin{aligned}
& \int_{C} d t^{\prime} \operatorname{sgn}_{C}\left(t-t^{\prime}\right)=\int_{t_{0}}^{t} d t^{\prime}-\int_{t}^{t_{0}} d t^{\prime}=2 \int_{t_{0}}^{t} d t^{\prime} \\
& \int_{C} d t^{\prime} \operatorname{sgn}_{C}\left(t-t^{\prime}\right) \operatorname{sgn}_{C}\left(t^{\prime}-t^{\prime \prime}\right)=2 \operatorname{sgn}_{C}\left(t-t^{\prime \prime}\right) \int_{t^{\prime \prime}}^{t} d t^{\prime}
\end{aligned}
$$

The RHS of Eq. (88) can be written in terms of $K$ and $\rho$ components by using the above identities. The first term is

$$
\begin{aligned}
& \frac{1}{2 i} \int d x^{\prime} d t^{\prime} \Sigma\left(x^{\prime} t^{\prime}, x t\right) \partial_{\mu} G\left(x t, x^{\prime} t^{\prime}\right)= \\
& \frac{1}{8 i} \int d x^{\prime} d t^{\prime}\left[\Sigma^{K}\left(x^{\prime} t^{\prime}, x t\right)-\Sigma^{\rho}\left(x^{\prime} t^{\prime}, x t\right) \operatorname{sgn}_{\mathrm{C}}\left(t^{\prime}-t\right)\right] \\
& \times\left[\partial_{\mu} G_{K}\left(x t, x^{\prime} t^{\prime}\right)-\partial_{\mu} G_{\rho}\left(x t, x^{\prime} t^{\prime}\right) \operatorname{sgn}_{C}\left(t-t^{\prime}\right)\right]
\end{aligned}
$$

where $G_{\rho}\left(x t, x^{\prime} t^{\prime}\right) \partial_{t} \operatorname{sgn}_{C}\left(t-t^{\prime}\right)=2 G_{\rho}\left(x t, x^{\prime} t^{\prime}\right) \delta_{C}(t-$ $\left.t^{\prime}\right)=0$ was used. Now using identities A1 and

$$
\begin{aligned}
& \int_{C} d t^{\prime} \Sigma^{K}\left(x^{\prime} t^{\prime}, x t\right) \partial_{\mu} G_{K}\left(x t, x^{\prime} t^{\prime}\right) \\
& =\int_{t_{0}}^{t_{\max }} d t^{\prime} \Sigma^{K}\left(x^{\prime} t^{\prime}, x t\right) \partial_{\mu} G_{K}\left(x t, x^{\prime} t^{\prime}\right) \\
& +\int_{t_{\max }}^{t_{0}} d t^{\prime} \Sigma^{K}\left(x^{\prime} t^{\prime}, x t\right) \partial_{\mu} G_{K}\left(x t, x^{\prime} t^{\prime}\right) \\
& =0
\end{aligned}
$$

we end up with two terms only

$$
\begin{aligned}
& \left.\frac{1}{8 i} \int_{-\Sigma^{\rho}} d x^{\prime} d t^{\prime}\left[-\Sigma^{\prime} t^{\prime}, x t\right) \partial_{\mu} G_{K}\left(x t, x^{\prime} t^{\prime} t^{\prime}\right) \operatorname{sgn}_{\mathrm{C}}\left(t^{\prime}-t\right)\right] \\
& =-\frac{1}{4 i}\left(\int_{0}^{t} d x^{\prime} \int_{0}^{t} d t^{\prime} \Sigma^{K}\left(x^{\prime} t^{\prime}, x t\right) \partial_{\mu} G_{\rho}\left(x t, x^{\prime} t^{\prime}\right)\right. \\
& \left.-\int d x^{\prime} \int_{0}^{t} d t^{\prime} \Sigma^{\rho}\left(x^{\prime} t^{\prime}, x t\right) \partial_{\mu} G_{K}\left(x t, x^{\prime} t^{\prime}\right)\right)
\end{aligned}
$$

Note that $t_{0}=0$ as the cosine potential and hence the self-energies are non-zero only after this time. The second term of Eq. (88) is

$$
\begin{aligned}
& \frac{1}{8 i} \int_{0} d x^{\prime} d t^{\prime}\left[-\Sigma^{K}\left(x t, x^{\prime} t^{\prime}\right) \partial_{\mu} G_{\rho}\left(x^{\prime} t^{\prime}, x t\right) \operatorname{sgn}_{C}\left(t^{\prime}-t\right)\right. \\
& \left.-\Sigma^{\rho}\left(x t, x^{\prime} t^{\prime}\right) \partial_{\mu} G_{K}\left(x^{\prime} t^{\prime}, x t\right) \operatorname{sgn}_{\mathrm{C}}\left(t-t^{\prime}\right)\right] \\
& =\frac{1}{8 i} \int d x^{\prime} d t^{\prime}\left[\Sigma^{K}\left(x^{\prime} t^{\prime}, x t\right) \partial_{\mu} G_{\rho}\left(x t, x^{\prime} t^{\prime}\right) \operatorname{sgn}_{C}\left(t^{\prime}-t\right)\right. \\
& \left.+\Sigma^{\rho}\left(x^{\prime} t^{\prime}, x t\right) \partial_{\mu} G_{K}\left(x t, x^{\prime} t^{\prime}\right) \operatorname{sgn} n_{\mathrm{C}}\left(t-t^{\prime}\right)\right] \\
& =-\frac{1}{4 i}\left(\int_{0}^{t} d x^{\prime} \int_{0}^{t} d t^{\prime} \Sigma^{K}\left(x^{\prime} t^{\prime}, x t\right) \partial_{\mu} G_{\rho}\left(x t, x^{\prime} t^{\prime}\right)\right. \\
& \left.-\int d x^{\prime} \int_{0}^{t} d t^{\prime} \Sigma^{\rho}\left(x^{\prime} t^{\prime}, x t\right) \partial_{\mu} G_{K}\left(x t, x^{\prime} t^{\prime}\right)\right)
\end{aligned}
$$

The terms in Eqs. (A4) and (A5) are equal, and summing them we obtain Eq. (89).

\section{Appendix B: Proof of Eq. (99)}

Here we will show that $\frac{d T_{\Sigma}^{00}(T)}{d T}=0$. Since from energy conservation $\frac{d E}{d T}=0$, this implies that $\frac{d T_{\mathrm{kin}}^{00}(T)}{d T}=0$. Using Eq. (97), we find that

$$
\begin{aligned}
& \frac{d T_{\Sigma}^{00}}{d T}=-\frac{u K}{4} \int_{0}^{\infty} d q e^{-\alpha q}\left[i \Sigma^{K}(q, T)\right. \\
& \left.-i\left(\Sigma^{R}-\Sigma^{A}\right)(q, T) F(q, T)\right]
\end{aligned}
$$

Using Eq. (54)

$$
\begin{aligned}
& \int_{0}^{\infty} d q e^{-\alpha q} i \Sigma^{K}=\left(\frac{g u \gamma}{\alpha^{2}}\right)^{2} \int_{-\infty}^{\infty} d \tau \int_{-\infty}^{\infty} d r \\
& \times e^{-I(T, r, \tau)} \cos \left[K_{\mathrm{eq}} \sum_{\varepsilon= \pm 1} \tan ^{-1}\left(\frac{u \tau+\varepsilon r}{\alpha}\right)\right] \\
& \times \int_{0}^{\infty} d q e^{-\alpha q} \cos (q r) \cos (u q \tau)
\end{aligned}
$$

Using $\quad \int_{0}^{\infty} d q e^{-\alpha q} \cos (q r) \cos (u q \tau) \quad=$ $\frac{1}{2 \alpha} \sum_{\epsilon= \pm} \frac{1}{1+(u \tau+\epsilon r)^{2} / \alpha^{2}}$ and that

$$
\begin{aligned}
& \frac{\alpha}{K_{\mathrm{eq}}} \frac{\partial}{\partial(u \tau+\epsilon r)} \sin \left[K_{\mathrm{eq}} \sum_{\epsilon^{\prime}= \pm} \tan ^{-1}\left(\frac{u \tau+\epsilon^{\prime} r}{\alpha}\right)\right]= \\
& \cos \left[K_{\mathrm{eq}} \sum_{\epsilon^{\prime}= \pm} \tan ^{-1}\left(\frac{u \tau+\epsilon^{\prime} r}{\alpha}\right)\right]\left[\frac{1}{1+(u \tau+\epsilon r)^{2} / \alpha^{2}}\right]
\end{aligned}
$$


we find,

$$
\begin{aligned}
& \int_{0}^{\infty} d q e^{-\alpha q} i \Sigma^{K}=\left(\frac{g u \gamma}{\alpha^{2}}\right)^{2} \int_{-\infty}^{\infty} d \tau \int_{-\infty}^{\infty} d r e^{-I(T, r, \tau)} \\
& \frac{1}{2 K_{\text {eq }}} \sum_{\epsilon^{\prime}= \pm} \frac{\partial}{\partial\left(u \tau+\epsilon^{\prime} r\right)} \sin \left[K_{\text {eq }} \sum_{\varepsilon= \pm} \tan ^{-1}\left(\frac{u \tau+\varepsilon r}{\alpha}\right)\right]
\end{aligned}
$$

Now one may integrate the above expression by parts and use that $\frac{\partial}{\partial(u \tau+\epsilon r)} I(r, \tau, T)=$ $K_{\text {eq }} \int_{0}^{\infty} d q e^{-\alpha q} F(q, T) \sin [q(u \tau+\epsilon r)] . \quad$ This leads to

$$
\begin{aligned}
& \int_{0}^{\infty} d q e^{-\alpha q} i \Sigma^{K}=\left(\frac{g u \gamma}{\alpha^{2}}\right)^{2} \int_{-\infty}^{\infty} d \tau \int_{-\infty}^{\infty} d r \\
& \times e^{-I(T, r, \tau)} \sin \left[K_{\mathrm{eq}} \sum_{\varepsilon= \pm} \tan ^{-1}\left(\frac{u \tau+\varepsilon r}{\alpha}\right)\right] \\
& \times \int_{0}^{\infty} d q e^{-\alpha q} \cos (q r) \sin (u q \tau) F(q, T) \\
& =\int_{0}^{\infty} d q e^{-\alpha q} i\left[\Sigma^{R}-\Sigma^{A}\right](q, T) F(q, T)
\end{aligned}
$$

Thus we have proved Eq. (99).

\section{Appendix C: Perturbative evaluation of the inelastic scattering rate}

In this section we discuss the inelastic scattering rate or dissipation defined in Eq. (108) for two cases, one is for small deviations of the system from thermal equilibrium at a temperature $T_{\text {eq }}, 56$ and the second is by doing perturbation theory for the quantum quench. $\underline{42,65}$

In equilibrium and finite temperature, $K_{\text {eq }}=K_{\text {neq }}$ and and the distribution function of the bosons is $\left\langle 2 b_{p}^{\dagger} b_{p}+\right.$ $1\rangle=\operatorname{coth} \frac{u|p|}{2 T_{\mathrm{eq}}}$. This leads to the following inelastic scattering rate at temperature $T_{\text {eq }}$, (in units of the cut-off)

$$
\begin{aligned}
& \eta_{T}=\left(\frac{\pi K}{2}\right) g^{2} \gamma^{2} \int_{-\infty}^{\infty} d r \int_{-\infty}^{\infty} d \tau \\
& \tau \sin \left[K_{\mathrm{eq}} \tan ^{-1}(\tau+r)+K_{\mathrm{eq}} \tan ^{-1}(\tau-r)\right] \\
& e^{-K_{\mathrm{eq}}\left[f_{T}(\tau+r)+f_{T}(\tau-r)\right]}
\end{aligned}
$$

where

$$
\begin{aligned}
& f_{T}(x)=\int_{0}^{\infty} d q e^{-q}[1-\cos (q x)] \operatorname{coth}\left(\frac{u q}{2 T_{\mathrm{eq}} \alpha}\right) \\
& =\ln \left(\sqrt{1+x^{2}}\right)+2 \ln \Gamma\left(1+\frac{\alpha T_{\mathrm{eq}}}{u}\right) \\
& -\ln \Gamma\left(1+\frac{\alpha T_{\mathrm{eq}}}{u}-i \frac{\alpha T_{\mathrm{eq}} x}{u}\right) \\
& -\ln \Gamma\left(1+\frac{\alpha T_{\mathrm{eq}}}{u}+i \frac{\alpha T_{\mathrm{eq}} x}{u}\right)
\end{aligned}
$$

and $\Gamma$ is the Gamma function. For $x \gg 1$

$$
f_{T}(x \gg 1)=\ln \left[\frac{u}{\alpha \pi T_{\mathrm{eq}}} \sinh \left(\frac{\pi \alpha T_{\mathrm{eq}} x}{u}\right)\right]
$$

From this it follows that the dissipation at finite temperature scales with temperature as follows,

$$
\eta_{T} \propto g^{2} \gamma^{2}\left(\alpha T_{\mathrm{eq}} / u\right)^{2 K_{\mathrm{eq}}-3}
$$

in agreement with Ref. $\underline{56}$ if we set $\gamma=2 \sqrt{2}$.

Let us now consider the case of the quantum quench. Using bare correlators $(g=0)$ for the expectation values $\langle\phi \phi\rangle$ (see Section IA ), explicit expressions for the selfenergy in Eqns. (36) and (37) are given below,

$$
\begin{aligned}
& \Pi^{R}\left(x_{1} t_{1}, x_{2} t_{2}\right)=-\left(\frac{g u \gamma}{\alpha^{2}}\right)^{2} \theta\left(t_{1}-t_{2}\right) \\
& \sin \left[K_{\mathrm{eq}} \tan ^{-1}\left(\frac{u\left(t_{1}-t_{2}\right)+\left(x_{1}-x_{2}\right)}{\alpha}\right)\right. \\
& \left.+K_{\mathrm{eq}} \tan ^{-1}\left(\frac{u\left(t_{1}-t_{2}\right)-\left(x_{1}-x_{2}\right)}{\alpha}\right)\right] \\
& \times f_{\mathrm{ss}}\left(x_{1}-x_{2}, t_{1}-t_{2}\right) f_{\mathrm{tr}}\left(x_{1}-x_{2}, t_{1}, t_{2}\right)
\end{aligned}
$$

and

$$
\begin{aligned}
& \Pi^{K}\left(x_{1} t_{1}, x_{2} t_{2}\right)=-i\left(\frac{g u \gamma}{\alpha^{2}}\right)^{2} \\
& \cos \left[K_{\mathrm{eq}} \tan ^{-1}\left(\frac{u\left(t_{1}-t_{2}\right)+\left(x_{1}-x_{2}\right)}{\alpha}\right)\right. \\
& \left.+K_{\mathrm{eq}} \tan ^{-1}\left(\frac{u\left(t_{1}-t_{2}\right)-\left(x_{1}-x_{2}\right)}{\alpha}\right)\right] \\
& \times f_{\mathrm{ss}}\left(x_{1}-x_{2}, t_{1}-t_{2}\right) f_{\mathrm{tr}}\left(x_{1}-x_{2}, t_{1}, t_{2}\right)
\end{aligned}
$$

where $f_{\mathrm{ss}}$ is a function that is translationally invariant in time, while $f_{\text {tr }}$ contains transients. Their explicit expressions are,

$$
\begin{aligned}
& f_{\mathrm{ss}}\left(x_{1}-x_{2}, t_{1}-t_{2}\right)=\left[\sqrt{\frac{\alpha^{2}}{\alpha^{2}+\left[u\left(t_{1}-t_{2}\right)+\left(x_{1}-x_{2}\right)\right]^{2}}} \quad(\mathrm{C} 7)\right. \\
& \times \sqrt{\left.\frac{\alpha^{2}}{\alpha^{2}+\left[u\left(t_{1}-t_{2}\right)-\left(x_{1}-x_{2}\right)\right]^{2}}\right]^{K_{\text {neq }}}} \\
& f_{\operatorname{tr}}\left(x_{1}-x_{2}, t_{1}, t_{2}\right)=\left[\sqrt{\frac{\alpha^{2}+\left(2 u t_{1}\right)^{2}}{\alpha^{2}+\left[u\left(t_{1}+t_{2}\right)+\left(x_{1}-x_{2}\right)\right]^{2}}}\right. \\
& \times \sqrt{\left.\frac{\alpha^{2}+\left(2 u t_{2}\right)^{2}}{\alpha^{2}+\left[u\left(t_{1}+t_{2}\right)-\left(x_{1}-x_{2}\right)\right]^{2}}\right]^{K_{t r}}} \quad
\end{aligned}
$$

Above $K_{\text {eq }}=\frac{\gamma^{2}}{4} K, K_{\text {neq }}=\frac{\gamma^{2}}{8} K_{0}\left(1+\frac{K^{2}}{K_{0}^{2}}\right)$, and $K_{\mathrm{tr}}=$ $\frac{\gamma^{2}}{8} K_{0}\left(1-\frac{K^{2}}{K_{0}^{2}}\right)$. At very long times after the quench, we may set $\left(t_{1}+t_{2}\right) / 2 \rightarrow \infty$ while keeping $t_{1}-t_{2}$ arbitrary. In this case $f_{t r} \rightarrow 1$ and the the scattering rates may be 
evaluated analytically to give,

$$
\begin{aligned}
& \eta_{0}=\left(\frac{\pi K}{2}\right) g^{2} \gamma^{2} \\
& \times\left[\frac{\pi}{2^{K_{\mathrm{neq}}-1}\left(K_{\mathrm{neq}}-1\right) B\left(\frac{K_{\mathrm{eq}}+K_{\mathrm{neq}}}{2}, \frac{K_{\mathrm{neq}}-K_{\mathrm{eq}}}{2}\right)}\right] \\
& \times\left[\frac{\pi}{2^{K_{\mathrm{neq}}-2}\left(K_{\mathrm{neq}}-2\right) B\left(\frac{K_{\mathrm{eq}}+K_{\mathrm{neq}}-2}{2}, \frac{K_{\mathrm{neq}}-K_{\mathrm{eq}}}{2}\right)}\right. \\
& \left.-\frac{\pi}{2^{K_{\mathrm{neq}}-2}\left(K_{\mathrm{neq}}-2\right) B\left(\frac{K_{\mathrm{eq}}+K_{\mathrm{neq}}}{2}, \frac{K_{\mathrm{neq}}-K_{\mathrm{eq}}-2}{2}\right)}\right]
\end{aligned}
$$

Another useful quantity is the strength of the noise which is given by the correlation function $42,65 \Pi^{K}$. Its expression at long-wavelengths is given by,

$$
\begin{aligned}
& I_{K}=i \int_{-\infty}^{\infty} d \tau \int_{-\infty}^{\infty} d r \\
& \times \Pi_{K}\left(r, \tau=t_{1}-t_{2}, \frac{t_{1}+t_{2}}{2} \rightarrow \infty\right)
\end{aligned}
$$

Within perturbation theory we obtain,

$$
\begin{aligned}
& I_{K}=2 g^{2} \gamma^{2} \times \\
& {\left[\frac{\pi}{2^{K_{\text {neq }}-1}\left(K_{\text {neq }}-1\right) B\left(\frac{K_{\text {eq }}+K_{\text {neq }}}{2}, \frac{K_{\text {neq }}-K_{\text {eq }}}{2}\right)}\right]^{2}}
\end{aligned}
$$

The ratio of the noise in Eq. (C11) and the dissipation in Eq. (C9) may be used to define an effective temperature,

$$
\tilde{T}_{\mathrm{eff}}=\frac{u}{\alpha}\left(\frac{K_{\mathrm{neq}}-2}{2 K_{\mathrm{eq}}}\right)
$$

This effective-temperature does not have any physical meaning as the system is out of equilibrium, and different combinations of response and correlation functions in general will give different effective-temperatures. However, it is interesting to note that in the limit of large quenches $K_{\text {neq }} \gg K_{\text {eq }}$, this temperature approaches the true temperature $T_{\text {eq }}$ (Eq. (26) ) the system would reach if it thermalized. 